Revue musicale OICRM

\title{
Essai de caractérisation de l'évolution des musiques super-héroïques de Batman (1989) à The Dark Knight Rises (2012)
}

\section{Jérôme Rossi}

Volume 5, numéro 2, 2018

Création musicale et sonore dans les blockbusters de Remote Control

URI : https://id.erudit.org/iderudit/1054146ar

DOI : https://doi.org/10.7202/1054146ar

\section{Aller au sommaire du numéro}

\section{Éditeur(s)}

Observatoire interdisciplinaire de création et recherche en musique (OICRM)

ISSN

2368-7061 (numérique)

Découvrir la revue

Citer cet article

Rossi, J. (2018). Essai de caractérisation de l'évolution des musiques super-héroïques de Batman (1989) à The Dark Knight Rises (2012). Revue musicale OICRM, 5(2), 15-47. https://doi.org/10.7202/1054146ar

\section{Résumé de l'article}

Vingt-trois années séparent les musiques des films Batman (Tim Burton, 1989) et The Dark Knight Rises (Christopher Nolan, 2012) avec les compositions respectives de Danny Elfman et Hans Zimmer. Si les compositeurs restent tous deux fidèles à une conception signalétique des thèmes, Zimmer propose toutefois un nombre plus élevé de matériaux thématiques, avec la présence d'un " ostinato identifiant " pour chacun des trois personnages principaux, en plus de leurs thèmes propres.

Élaborée par une véritable équipe, la « narration sonore » constitue également un enjeu majeur de l'esthétique de Nolan et Zimmer avec l'élaboration, sur l'ensemble du film, d'un continuum bruit/musique, dont ce que nous avons appelé l'" effects underscoring » constitue l'une des stratégies les plus novatrices parmi celles proposées. L'« effects underscoring » est une technique compositionnelle par laquelle la musique se voit destinée à former un écrin émotionnel non plus aux voix, mais aux bruits lors de séquences où ce sont eux qui, par leur propriétés intrinsèques (organisation rythmique, caractéristiques timbrales), concentrent la signification dramaturgique d'un passage. Le travail minutieux sur les bruits, qui s'ajoute à une meilleure définition de ceux-ci grâce à la technologie numérique, « libère " la musique dans son rapport à l'image ; nous en observons les effets concrets en comparant les synchronismes et le maniement des ostinatos (longueur, pédales harmoniques, rythme harmonique) dans des scènes d'action empruntées aux deux films. Tandis que le travail d'Elfman se caractérise par une écriture vive et prompte à souligner tant les actions des personnages que les changements de plans, Zimmer se détache des évènements visuels en privilégiant une plus grande continuité musicale qui repose en partie sur ce nous avons appelé le stem scoring, soit une composition musicale conçue en plusieurs strates que le compositeur ou le mixeur peut à loisir déclencher ou taire en fonction de l'image.
Ce document est protégé par la loi sur le droit d'auteur. L’utilisation des services d’Érudit (y compris la reproduction) est assujettie à sa politique d'utilisation que vous pouvez consulter en ligne.

https://apropos.erudit.org/fr/usagers/politique-dutilisation/ 


\title{
Essai de caractérisation de l'évolution des musiques super- héroïques de Batman (1989) à The Dark Knight Rises (2012)
}

\author{
Jérôme Rossi
}

\begin{abstract}
Résumé
Vingt-trois années séparent les musiques des films Batman (Tim Burton, 1989) et The Dark Knight Rises (Christopher Nolan, 2012) avec les compositions respectives de Danny Elfman et Hans Zimmer. Si les compositeurs restent tous deux fidèles à une conception signalétique des thèmes, Zimmer propose toutefois un nombre plus élevé de matériaux thématiques, avec la présence d'un " ostinato identifiant " pour chacun des trois personnages principaux, en plus de leurs thèmes propres.

Élaborée par une véritable équipe, la " narration sonore " constitue également un enjeu majeur de l'esthétique de Nolan et Zimmer avec l'élaboration, sur l'ensemble du film, d'un continuum bruit/musique, dont ce que nous avons appelé l'« effects underscoring " constitue l'une des stratégies les plus novatrices parmi celles proposées. L' ' effects underscoring» est une technique compositionnelle par laquelle la musique se voit destinée à former un écrin émotionnel non plus aux voix, mais aux bruits lors de séquences où ce sont eux qui, par leur propriétés intrinsèques (organisation rythmique, caractéristiques timbrales), concentrent la signification dramaturgique d'un passage. Le travail minutieux sur les bruits, qui s'ajoute à une meilleure définition de ceux-ci grâce à la technologie numérique, « libère » la musique dans son rapport à l'image ; nous en observons les effets concrets en comparant les synchronismes et le maniement des ostinatos (longueur, pédales harmoniques, rythme harmonique) dans des scènes d'action empruntées aux deux films. Tandis que le travail d'Elfman se caractérise par une écriture vive et prompte à souligner tant les actions des personnages que les changements de plans, Zimmer se détache des évènements visuels en privilégiant une plus grande continuité musicale qui repose en partie sur ce nous avons appelé le stem scoring, soit une composition musicale conçue en plusieurs strates que le compositeur ou le mixeur peut à loisir déclencher ou taire en fonction de l'image.
\end{abstract}

Mots clés : musique de film ; bruits ; effects underscoring; stem scoring; rythme harmonique.

\footnotetext{
Abstract

Twenty-three years separate the music from Batman (Tim Burton, 1989) and The Dark Knight Rises (Christopher Nolan, 2012) with the respective compositions of Danny Elfman and Hans Zimmer. If both composers remain faithful to a signifying
} 
design of the themes, Zimmer proposes a higher number of thematic materials, with the presence of an "identifying ostinato" for each of the three main characters, in addition to their own themes.

Developed by a real team, "sound narration" is also a major characteristic of Nolan and Zimmer's aesthetics with the elaboration, throughout the film, of a continuum of sound effects and music, in which what we've called "effects underscoring" constitutes one of the most innovative strategies. "Effects underscoring" is a compositional technique by which music is intended to create an emotional setting no longer to the voices, but to the noises, during sequences where sounds concentrate the dramaturgical meaning of a section through their intrinsic properties (rhythmic organization, timbral characteristics) The meticulous work on noises, adding to a better definition of sound effects thanks to digital technology, really "frees" music in its relationship to the image; we observe the concrete effects of this by comparing the synchronisms and the handling of the ostinatos (length, harmonic pedals, harmonic rhythm) in action scenes from the two films. While Elfman's work is characterized by a vivid and detailed writing which emphasizes both the characters' actions and the editing, Zimmer distances himselfs from visual events by privileging a greater musical continuity which mostly depends on what we have called "stem scoring", a multi-layered musical composition that the composer or mixer can trigger or remove, depending on the image.

Keywords: film music; sound effects; effects underscoring; stem scoring; harmonic rhythm.

Si Danny Elfman et Hans Zimmer viennent tous deux de la pop music et du rock, origines qui se traduisent par de fréquents enrichissement de l'orchestre symphonique à l'aide de timbres électrifiés ou synthétiques, leurs styles musicaux demeurent assez éloignés. Collaborant depuis ses premiers films avec Tim Burton, le premier est associé à une combinaison d'éléments obscurs et étranges ; tonalités mineures, colorations lydiennes, mélodies anguleuses, tempi élevés, goût pour les timbres instrumentaux purs et les chœurs, sont quelques-uns des marqueurs singuliers elfmanniens que l'on retrouve dans Scrooged (R. Donner, 1988), To Die For (G. van Sant, 1995), Freeway (M. Bright, 1996), The Frighteners (P. Jackson, 1996) et A Simple Plan (S. Raimi, 1998), comme dans la totalité des films de Tim Burton à l'exception de Planet of the Apes (2001). De son côté, Hans Zimmer s'est d'abord concentré sur les sonorités synthétiques avec sa première partition hollywoodienne pour Rain Man (B. Levinson, 1988) avant d'intégrer progressivement l'orchestre symphonique à sa palette tout en conservant une forte assise électronique. Au sein des deux structures qu'il a successivement créées - Media Ventures en 1989 puis Remote Control Production en 2003 -, Zimmer s'est entouré de compositeurs, d'ingénieurs du son et d'interprètes, lui permettant de se concentrer sur la partie la plus créative :

Hans is the master of investing fully in the ideas of a film more than the details. By having orchestration help, arranging help, you're able to back a bit and have an objective view that you could otherwise never have (Burlingame 2014).

Le nom de Hans Zimmer est associé aux grands succès du box-office de ces dernières années : The Lion King (R. Allers et R. Minkoff, 1995), Gladiator (R. Scott, 
2000), Pirates of the Caribbean: Dead Man's Chest (G. Verbinski, 2006), The Da Vinci Code (R. Howard, 2006), Batman Begins (C. Nolan, 2005), Pearl Harbor (M. Bay, 2011). Zimmer a collaboré de manière récurrente avec Ron Howard, Gore Verbinski, et Christopher Nolan ${ }^{1}$.

En prenant pour corpus les compositions pour Batman (T. Burton, 1989) ${ }^{2}$ de Danny Elfman et de The Dark Knight Rises (C. Nolan, 2012) ${ }^{3}$ de Hans Zimmer, soit deux films réalisés à vingt-trois ans d'intervalle ${ }^{4}$, nous nous poserons la question tout en gardant à l'esprit que les films eux-mêmes sont très différents - de l'évolution de l'écriture musicale dans les bandes originales des films de super-héros, un corpus de plus en plus questionné tant par les études en cinéma (Page 2007, Jess-Cooke 2009, Brooker 2012) que par la littérature musicologique (Halfyard 2004, Halfyard 2013, Solis 2013, Hexel 2016, Lehman 2017).

Notre attention se portera successivement sur les caractérisations thématiques de Batman et de ses ennemis - le Joker (Jack Nicholson) dans le premier film, Bane (Tom Hardy) dans le second - ainsi que sur les personnages secondaires, le travail sur les bruits (sound design) - plus important dans The Dark Knight Rises de Nolan - et enfin le rapport rythmique de ces musiques par rapport aux images dans les scènes d'action des deux films.

\section{MATÉRIAUX THÉMATIQUES}

\section{Thématique super-héroïque}

À la fin des années soixante-dix et pendant les années quatre-vingt, John Williams a incontestablement façonné l'imaginaire musical des super-héros au cinéma ${ }^{5}$

1 Avant de confier la partition de Batman Begins à Zimmer et James Newton Howard (voir infra), Nolan a travaillé avec David Julyan sur Following (1998), Memento (2000), Insomnia (2002) et The Prestige (2005). La raison de l'éviction de Julyan de Batman Begins semble devoir être attribuée à un choix de la part de Warner Brothers (Hexel 2017, p. 9).

2 Les exemples vidéo donnés s'appuient sur la VF du DVD Warner Home Video ; les exemples musicaux sont tirés de la BO parue chez Warner Bros Records (1989).

3 Les exemples vidéo donnés s'appuient sur la VF du DVD Warner Home Video ; les exemples musicaux sont tirés de la BO parue chez Sony Classical (2012).

4 Après la série télévisée de 1966, Batman est le premier film d'une première tétralogie consacrée au héros de DC Comics, comprenant deux films de Tim Burton (Batman et Batman Returns) et deux de Joël Schumacher (Batman Forever et Batman and Robin); les musiques des deux premiers sont de Danny Elfman, les deux derniers ont des partitions d'Elliot Goldenthal. Batman: The Dark Knight Rises clôt une nouvelle trilogie comprenant également Batman Begins (C. Nolan, 2005) et Batman: The Dark Knight (C. Nolan, 2007). La musique des deux premiers films a été composée par James Newton-Howard et Hans Zimmer, tandis que le troisième volet est entièrement signé par ce dernier. Une partie de la musique de The Dark Knight Rises provient des deux premiers opus. Selon Zimmer : "We worked really hard at creating this sort of sonic world which is Gotham. You don't want to go and suddenly break out of that. I very deliberately kept the sonic landscape " (Hyman 2012).

$5 \quad$ Philip Tagg a montré que le topique héroïque est majoritairement associé à la masculinité et aux représentations militaires (Tagg 1982 ; Tagg 1989). Avant John Williams, ce topique était déjà utilisé dans les films d'aventure et les westerns. 
à travers ses compositions pour la première trilogie Star Wars (G. Lucas, 1977 ; I. Kershner, 1981 ; R. Marquand, 1983), la tétralogie des Indiana Jones ${ }^{6}$ (S. Spielberg, 1981, 1984, 1989, 2008) ou encore le premier Superman (R. Donner, 1978). Pour ce dernier, Williams a conçu un thème de profil ascendant, confié aux cuivres sur des ostinatos réguliers de cordes, énoncé sur un tempo énergique et une rythmique martiale $^{7}$ (prédominance de la figure croche pointée/double croche) marquée aux timbales, tout cela soutenu par un schéma harmonique fermement ancré dans la tonalité (Rossi 2011).

De ce point de vue, le thème de Batman (Michael Keaton) composé par Danny Elfman marque une forme de rupture dans la continuité. Également énoncé aux cuivres, le thème s'appuie sur un rythme de marche militaire ; mais d'abord exposé en mineur (exemple 1), le thème - qu'il serait plus juste de qualifier de " motif » en raison de sa brièveté - fait ensuite l'objet de nombreuses modulations, au sein desquelles le compositeur évite systématiquement toute résolution dominante/ tonique ; à l'héroïque diatonisme et à la monotonalité, Elfman préfère le clair-obscur du chromatisme et les nombreuses modulations.

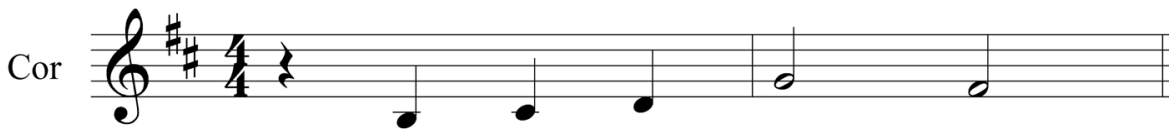

Exemple 1: Danny Elfman, Batman, cue "Batman Theme", motif de Batman. Transcription personnelle à partir de la bande originale, 00:00-00:09.

Extrait audio 1: Danny Elfman, Batman, cue "Batman Theme", motif de Batman. Bande originale, 00:00-00:09. Écouter.

Si cet écart par rapport à la «norme williamsienne » trouve sa justification narrative dans le caractère ambivalent et complexe du personnage de Batman, il n'en a pas moins été l'objet de discussions avec Jon Peters, l'un des producteurs :

I had written all this dark music, and Jon Peters was saying "Look, this is fine, but you know, we're talking about a hero here!" I played him all these pieces... but, at this point, it was essential that I came up with this one heroic theme. I just took the same basic theme and turned it into this march, and did it a certain way - changed the key a little bit - and all of sudden [Jon Peters, the producer] leapt up out of his chair and it was completely obvious that I has found the Batman hero theme (Halfyard 2013, p. 27).

Danny Elfman a certainement fait évoluer la conception musicale des super-héros vers une tendance moins positive, une direction suivie ensuite par Elliot Goldenthal, l'auteur des musiques des troisième et quatrième Batman.

À un motif immédiatement reconnaissable, le Batman (Christian Bale) de Nolan, Zimmer et Howard se voit affecté plutôt une simple tierce mineure ré-fa - issu de

6 Indiana Jones n'est pas un super-héros, car son but n'est pas de combattre le mal à l'aide de superpouvoirs ; toutefois son statut dans les films peut le rattacher à cette lignée : invincibilité, actions spectaculaires, double personnalité (le professeur Henry Jones Jr. et l'aventurier).

7 Pour John Williams, le goût pour les marches relève d'une forme de nostalgie (Colburn 2004). 
l'incipit d'un thème plus long (exemple 6) -, toujours confiée aux cuivres et souvent superposée à un ostinato rythmique en doubles croches (96 bpm), joué détaché aux cordes graves (renforcées par des samples), démultipliant cette même tierce mineure et pouvant facilement s'adapter à des contextes harmoniques changeants (exemple 5); cet « ostinato identifiant » peut être découplé de la tierce mineure emblématique.

On peut repérer trois harmonisations principales de la tierce mineure, associées à des significations différentes :

- la stase sur ré mineur (exemple 2), avec seulement un changement de renversement, connote l'attente, par exemple lorsque Wayne s'apprête à revêtir son costume de Batman;

- le glissement chromatique de ré mineur à do\# mineur (exemple 3, avec enharmonie du fa et du mi\#) renvoie au danger, par exemple lorsque Wayne est poursuivi par les policiers (01:47:30-01:47:38);

- la progression de ré mineur sur sib majeur (exemple 4) est le symbole de la réussite, lorsque Wayne parvient à sortir du puits (01:57:00-01:57:10).

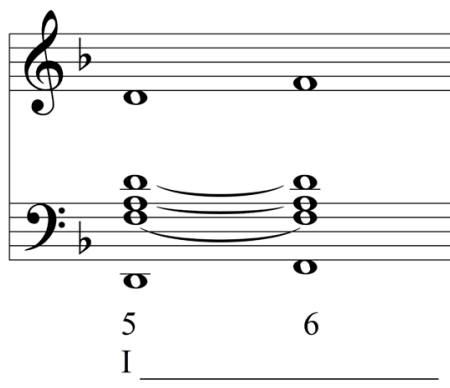

Exemple 2 : Hans Zimmer, The Dark Knight Rises, harmonisation du motif de Batman sur I. Transcription personnelle à partir du film, 01:04:55-02:05:10.

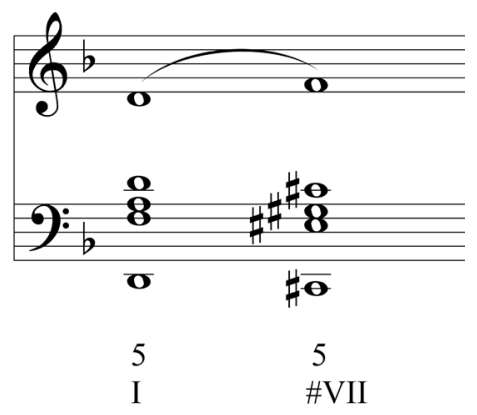

Exemple 3 : Hans Zimmer, The Dark Knight Rises, harmonisation du motif de Batman sur I-\#VII. Transcription personnelle à partir du film, 01:47:30-01:47:38.

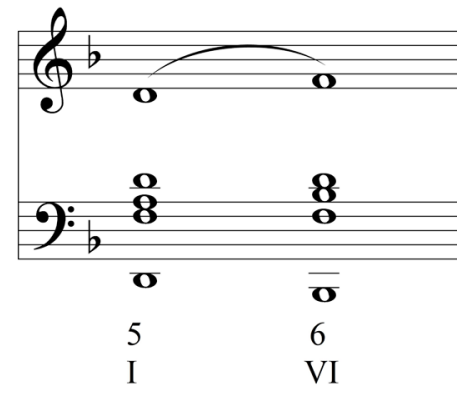

Exemple 4 : Hans Zimmer, The Dark Knight Rises, harmonisation du motif de Batman sur I-VI. Transcription personnelle à partir du film, 01:57:00-01:57:10. 
En dehors du logo Warner au début du film, la tierce mineure mélodique est entendue pour la première fois à 26:05 lorsque le jeune John Blake (Joseph Gordon-Levitt) vient rencontrer Bruce Wayne pour le convaincre de réveiller le Batman ; 1'ostinato, lui, se fait entendre dès 07:33 lorsque le nom de Wayne est évoqué par l'un des convives de la fête organisée par Wayne Entreprises (exemple 5).

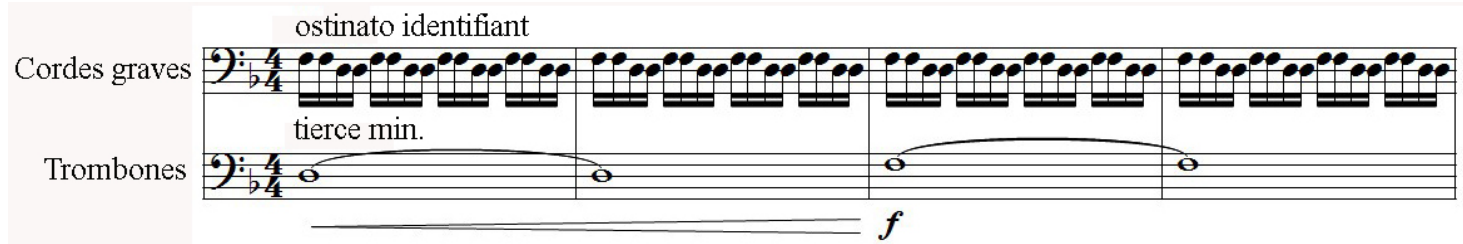

Exemple 5 : Hans Zimmer, The Dark Knight Rises, cue "Main Theme», tierce mineure de Batman superposée à l'ostinato identifiant. Transcription personnelle à partir de la bande originale, 00:18-00:30.

Extrait audio 2: Hans Zimmer, The Dark Knight Rises, cue "Main Theme", bande originale, 00:18-00:30. Écouter.

En un figuralisme sans équivoque, cette tierce, extrêmement présente pendant tout le film, reviendra par deux fois à la toute fin du générique de fin (02:43:47-02:44:30) dans un registre suraigu - alors qu'elle n'est entendue que dans le registre medium/ grave tout au long du film -, lorsqu'on voit s'afficher le titre du film, "The Dark Knight Rises » : Blake, que l'on a vu s'entraîner dans la Batcave dans les derniers plans, assume désormais son identité de «Robin » et va sans doute devenir lui-même Batman si le besoin s'en fait sentir. La première occurrence reste en ré mineur (" attente "), tandis que la seconde évolue sur le sixième degré (sib majeur), harmonie sur laquelle se termine le film en une cadence à la fois irrésolue et optimiste (" réussite ») : Batman va renaître en la personne de Robin et continuer de veiller à la sécurité des habitants de Gotham.

À l'occasion d'une interview, Newton Howard s'est expliqué sur cette volonté de s'éloigner des thèmes super-héroïques traditionnels : "When you write a traditional, conventional superhero theme, it gives you so much information that it might be misleading about that character. Our Batman? We're still getting to know him. He's a very complicated guy " (Newton Howard, cité dans Pigeon 2008). La brièveté motivique permet de nombreuses variations, une vertu utile dans le cas d'une trilogie : "When we did Batman Begins, we never thought we were going to spread two notes over three movies! The character changes and it's amazing how flexible two notes can become " (Newton Howard, cité dans Wayner 2014).

À côté de ce matériau peu caractérisé mélodiquement, Janet K. Halfyard a identifié dans Batman Begins un réseau de quatre thèmes secondaires (Halfyard 2013, p. 188) dont on n'entend parfois que l'accompagnement harmonique - qui reviennent périodiquement accompagner des actions du héros. L'un des plus importants est présent dès le début de la trilogie, lorsque les parents de Bruce se font tuer sous ses yeux (14:30), ou qu'il se souvient de leur assassinat (22:56) ; ce thème revient à la toute fin de The Dark Knight Rises, alors que l'on pense que Batman est mort : 


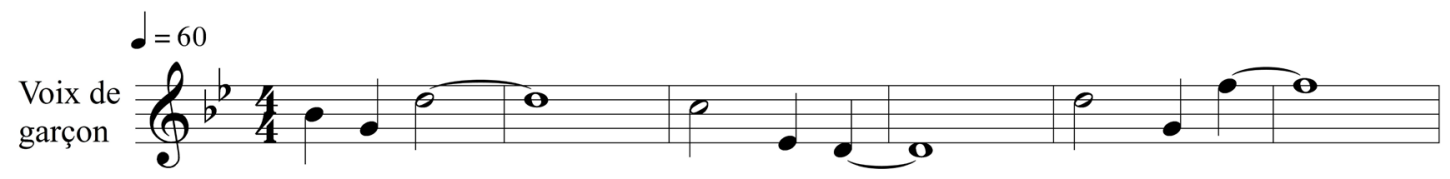

Exemple 6: Hans Zimmer, The Dark Knight Rises, «thème psychanalytique» de Batman. Transcription personnelle à partir du film, 02:30:50-02:31:15.

Extrait audio 3 : Hans Zimmer, The Dark Knight Rises, "thème psychanalytique » de Batman. Extraction audio à partir du film, 02:30:50-02:31:15. Écouter.

Peu commenté par les analystes, ce thème mérite pourtant que l'on s'y arrête. Anguleux, avec des intervalles disjoints, sans soubassement harmonique, rythmiquement peu affirmé, ce thème est aux antipodes d'un thème de super-héros. Zimmer en donne la signification, en l'occurrence psychanalytique :

If there was an arc to be had, it was that he sees his parents getting killed - that's the defining moment of his life - and he feels guilty for it. The theme is interrupted at essentially two notes: it's never completed, because he never gets past this point. I spent three weeks removing notes from the main Batman theme until I got it down to just two notes. I am very proud of it. It's always the beginning of a theme. It promises that he can become a hero or a complete man, but none of that ever happens, because he can't get past that moment (Zimmer, cité dans Wayner 2014).

La tierce mineure, signature motivique de Batman, proviendrait donc de l'incipit de ce "thème psychanalytique » et constituerait la partie la plus irréductible de la personnalité de Wayne/Batman ; son sacrifice à la fin de The Dark Knight Rises lui permet d'achever la mission de ses parents et de réaliser pleinement ce à quoi il était destiné, ce que corrobore la citation complète du thème à la toute fin de la trilogie.

La multiplicité de la thématique et son aspect peu caractérisé peuvent aussi être reliés, selon une intéressante hypothèse de Halfyard, à un choix visuel de la part du réalisateur :

This process of disguising the theme has a direct correspondence in the way Christopher Nolan constructs his Batman. In the DVD documentary Shaping Mind and Body, Nolan points out that in the action sequences we often do not see Batman clearly, because of the director's desire to present things from the criminal point of view. Batman appears and disappears with alarming speed, and we - like the criminals - often get only fleeting strains of the heroic theme; the music spends much of the time anticipating it via the harmonic progression, but rarely allows us to hear it clearly (Halfyard 2013, p. 190).

Opposition avec les super-vilains

Chaque film de Batman oppose le super-héros à un ennemi, voire plusieurs. Dans Batman, Danny Elfman a doté le super-héros d'un motif extrêmement caractérisé permettant une reconnaissance simple et rapide du spectateur. De son côté, le Joker n'a aucun thème propre, mais il est majoritairement associé - tant sous sa première forme de Jack Nappier que sous sa renaissance en Joker - à une 
texture inquiétante ayant recours au mode hexatonique (gamme par tons) ${ }^{8}$ et à des timbres symphoniques peu usuels (vibraphone, harmoniques de harpes, piano dans le registre grave, cordes utilisées avec un type de jeu particulier - pizzicato, glissando, sul ponticello ou sul tasto). Halfyard remarque que, dans la première partie du film (sur les trois distinguées par l'auteure), il y a une confusion possible entre Nappier et Batman. Ce dernier est présenté avec une musique hexatonique lors de son premier combat ("Roof Fight »), et son thème est présent au basson et aux cordes graves dans le repère de Grissom et Jack ("Card Snap »). Dans le cue "Shootout », le thème de Batman est même cité très brièvement sur le mode hexatonique (Halfyard 2004, p. 91). Pour l'auteure, cela signifie que Batman et Jack Nappier ont un point commun : ils évoluent tous deux dans un monde parallèle à la police. Mais, alors que Batman possède un thème, le médiocre Jack n'a pas de matériau thématique propre. De manière extrêmement intéressante, Halfyard montre que si l'acte 2 a dissipé toute association entre le mode hexatonique et Batman, l'acte 3 rétablit des connexions : Batman a pris conscience que Jack Nappier était le meurtrier de ses parents et il va agir - en partie - par vengeance : cette conduite le rapproche des voyous qui ne pensent qu'à leurs intérêts (cues " Batwing IIA » et " Showdown A »).

Un rythme particulier est également associé au Joker : la valse. C'est sur un rythme de valse qu'il révèle sa nouvelle identité en tuant Grissom (37:55), qu'il fait sa première déclaration en public en détournant d'ailleurs le thème de Batman en une version hexatonique (49:25), qu'il tombe amoureux de Vicky («Beautiful Dreamer ", 53:20-54:19), qu'il lui déclare ensuite sa flamme (01:24:35) et qu'il affronte Batman (01:51:00). Enfin, trois chansons de Prince appartiennent également à l'univers du Joker : Electric Chair, Partyman et Trust. Comme le résume Halfyard :

Overall, one of the most "features" of the musical representation of the Joker is the fragmentary and diverse nature of the music associated with him, with the use of unusual timbres and of four quite different genres; the Strauss waltz, "Beautiful dreamer" and the easy-listening idiom, the whole-tone music, and the Prince songs. This is itself an indication of the instability and irrationality of the Joker's character - it refuses to cohere, instead accumulating multiple identifications. The Joker, as his names indicates, is a wild card, unpredictable, and therefore difficult to pin down or contain (Halfyard 2013, p. 109).

Tout comme le Joker possède son rythme propre - la valse -, le super-vilain Bane a été doté par Zimmer d'un " ostinato identifiant » de type rythmique avec une mesure à $5 / 4$, que les accents divisent en $2+2+3+3$ :

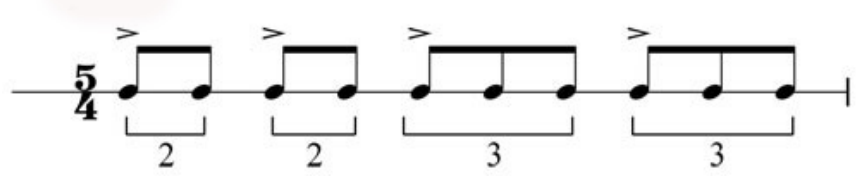

Exemple 7 : Hans Zimmer, The Dark Knight Rises, cue "Gothams Reckoning", ostinato de Bane. Transcription personnelle à partir de la bande originale, 02:45-02:47. 
Extrait audio 4 : Hans Zimmer, The Dark Knight Rises, cue "Gothams Reckoning ", ostinato de Bane. Bande originale, 02:45-02:47. Écouter.

On entend ce rythme dès les premières secondes du film aux cordes graves sur la même note ; lorsque le visage de Bane apparaît (03:24), le rythme est amplifié par des tambours graves de type taïko, tandis que des tenues de cuivres dans les graves génèrent un inquiétant suspense. Aux mots de Bane, «Détruire cet avion » (04:05), des clusters de cordes (registre medium) viennent renforcer les accents et épaissir la texture (par "stem scoring" voir infra). Les cordes dessinent des mouvements ascendants rapides de plus en plus dissonants ; lors de la transfusion, il ne reste plus que les tambours (05:26) avant que ne se joigne, à 05:55, un chœur d'hommes scandant les mots " Deschi Basara ", qui signifie "Relève-toi ! $»^{9}$. Désormais, la rythmique à $5 / 4$ sera associée à Bane, tandis que le motif de Batman reste fermement inscrit dans des mesures à 4/4. Après la prise d'otage à la bourse s'ensuit une course-poursuite où l'on voit Bane et ses hommes s'enfuir en moto (voir structure sonore de la scène en figure 1) ; Bane a la situation en main et l'ostinato qui se met en place est sur le modèle à $5 / 4$. Plus tard, quand les policiers s'engagent dans les tunnels de Gotham selon le plan de Bane, c'est encore le rythme de ce dernier qui résonne (01:23:15-01:24:00).

L'opposition entre Batman et Bane se joue ainsi d'abord sur un plan purement rythmique. Lorsqu'à 44:32 Batman arrive sur sa moto, la mesure se stabilise à 4/4, signifiant que c'est désormais le super-héros qui contrôle les événements. On retrouve cette opposition lorsque Batman sauve Selina Kyle de Bane (54:03, figure 3) ou lors de l'affrontement des partisans de Bane avec les policiers de Gotham (02:05:55) ; dans cette dernière séquence, le rythme est d'abord à $5 / 4$ jusqu'à l'arrivée de Batman (02:09:14). Notons que les deux rythmes peuvent aussi se superposer. Lorsque Wayne escalade le puits pour parvenir à la surface, il est par deux fois accompagné par le chant " Deschi Basara " à 5/4 : il se retrouve alors confronté à ses échecs comme l'avait prévu Bane. Lors de sa dernière tentative (01:56:42), qui sera fructueuse, le chant à $5 / 4$ est superposé au rythme binaire qui finit par dominer ; la tierce de Batman est alors entendue dans sa variante harmonique " réussite " tandis qu'un plan large sur un paysage désertique fait comprendre l'ampleur de la tâche qui attend Batman. Comme chez Elfman, l'opposition rythmique entre les deux personnages se double d'une opposition timbrale : le rythme de Bane est scandé par des incantations vocales, des tambours, des clusters de violons, c'est-à-dire des sonorités bruitistes et primitives, tandis que celui de Batman correspond à une matière sonore plus « civilisée »-fusion de matériaux acoustiques et électroniques.

Comme c'était le cas du «thème psychanalytique » de Batman, Bane a également un thème lié à son passé (exemple 8) ; comme nous l'apprendra la "légende du

9 À cette occasion, Hans Zimmer a sollicité ses fans pour participer à ce chant déclamatoire. En novembre 2011, il est passé par les réseaux sociaux (Twitter et Social Media) pour demander aux spectateurs de s'enregistrer en train de déclamer "Deschi Basara ». Selon Zimmer : "It seemed like an interesting thing. We've created this world, over these last two movies, and somehow I think the audience and the fans have been part of this world. We do keep them in mind. " (cité dans Radish 2011). 
mercenaire ${ }^{10}$, il est le protecteur de Miranda Tate, fille de Ra's al Ghul, que ce dernier a pris sous sa protection avant, finalement, de l'excommunier de la Ligue des ombres. On entend son thème pour la première fois joué aux cordes graves lorsqu'Alfred nous renseigne sur les origines de Bane (39:27) ; ce thème se parera ensuite de couleurs orientales jouée par une flûte orientale au fur et à mesure que la "légende " sera complétée (01:22:56, 01:42:05), jusqu'à la révélation finale (02:17:48). La connexion musicale entre ce thème - du moins son incipit - et la rythmique à $5 / 4$ de Bane a lieu entre l'évocation de la légende et la réquisition de la bombe (01:23:15) lorsque Zimmer propose une transition entre l'évocation de la légende et la réquisition de la bombe (01:23:15).

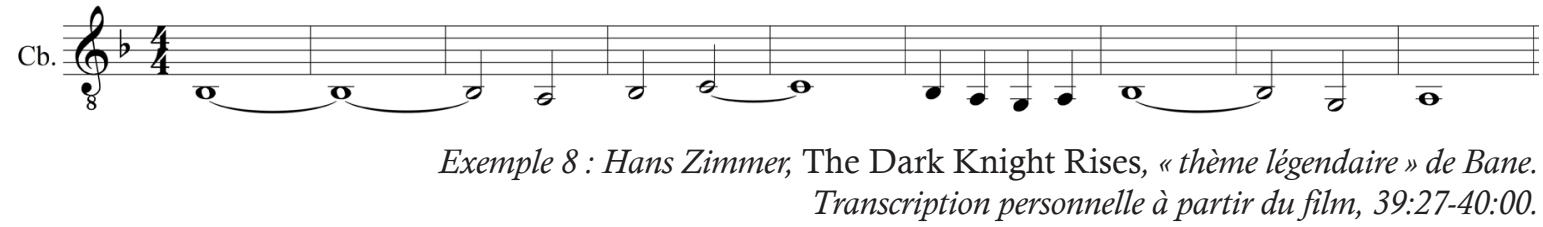

\section{Thématique secondaire}

Chez Elfman comme chez Zimmer, des thèmes complémentaires viennent enrichir les significations de certaines scènes par leurs récurrences à des moments clés.

Le premier propose un " thème du secret et des révélations " (Halfyard 2004, p. 120-122) et un «thème du destin » (ibid., p. 122-123) attachés à Batman qui restent toutefois très peu utilisés, ainsi que deux " thèmes d'amour ", choisis en fonction de l'amant considéré - le Joker ou Batman. Concernant le personnage féminin de Vicky Vale (Kim Basinger), Elfman ne donne qu'une coloration timbrale (le hautbois et la flûte dans « Batcave ») lorsque la jeune femme apparaît à l'écran.

De son côté, Zimmer, comme pour Batman et Bane, dote Selina Kyle (Anne Hathaway) à la fois d'un élément mélodique (exemple 9) et d'un " ostinato identifiant» (exemple 10) qui l'accompagne parfois, mais peut, comme celui de Batman, être utilisé seul. C'est l'occasion de remarquer combien ce compositeur peut se révéler extrêmement adroit dans le portait physique et psychologique d'un personnage. Le thème de Kyle est un élégant thème joué au piano qui traduit la beauté (timbre pur du piano) et la vivacité (présence de triple croches, chromatismes) du personnage, ainsi que sa complexité (mouvements disjoints à la fin) et son ambivalence - le thème se maintient sur la quinte de l'accord de ré mineur, référence à la position ambiguë de Kyle qui livre d'abord Batman à Bane avant finalement de rejeter le second pour s'associer au premier.

10 L'histoire de Bane est d'abord présentée à Wayne par Alfred comme une « rumeur »; elle est ensuite qualifiée de « vieille fable » et enfin de « légende » par le surveillant de Wayne dans la prison de Bane. 


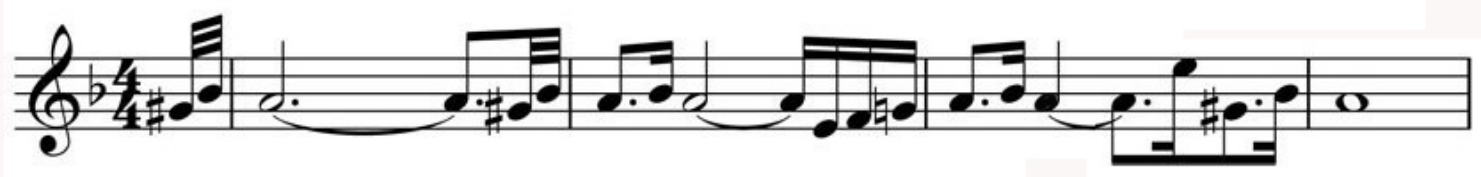

Exemple 9: Hans Zimmer, The Dark Knight Rises, cue "Mind if I cut in", thème de Selina Kyle. Transcription personnelle à partir de la bande originale, 00:44-00:59.

Extrait audio 5 : Hans Zimmer, The Dark Knight Rises, cue "Mind if I cut in ", thème de Selina Kyle. Bande originale, 00:44-00:59. Écouter.

L'« ostinato identifiant " de Kyle est à mettre en relation plus directe avec les actions du personnage ; sa construction en paliers successifs montre à la fois l'agilité et les limites de la jeune femme : la courte montée ne mène finalement nulle part, et l'ostinato en revient toujours à son début, symbole du personnage sans cesse rattrapé par son passé (la jeune femme cherche désespérément à se procurer le programme «Table Rase » pour effacer ses actions anciennes).

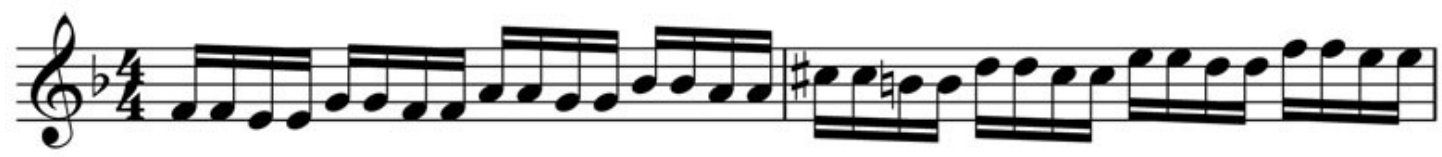

Exemple 10 : Hans Zimmer, The Dark Knight Rises, cue "Mind if I cut in ", ostinato de Selina Kyle. Transcription personnelle à partir de la bande originale, 02:08-02:11.

Extrait audio 6: Hans Zimmer, The Dark Knight Rises, cue "Mind if I cut in ", ostinato de Selina Kyle. Bande originale, 02:08-02:11. Écouter.

Les autres thèmes ne sont assurément pas du côté de l'héroïsme, mais plutôt du côté de l'intime et de la mort. On distingue une suite harmonique de trois accords (Dm, Dm/C, G) associée aux enfants orphelins, que l'on trouve dans une instrumentation synthétique (18:55, 27:44) ou symphonique (01:07:50, 01:08:48), selon qu'il s'agit de l'orphelinat ou de la propre histoire de Wayne. Deux thèmes, enfin, prennent leur sens dans l'ensemble de la trilogie. Le premier est celui de la ligue des ombres (01:44:28), lorsque Wayne a une hallucination en voyant Ra's al Ghul (Liam Neeson) apparaître dans la prison de Bane. L'autre thème consiste en réalité en deux motifs connotant le deuil et l'affliction - Hexel le nomme "mourning " ${ }^{11}$ (Hexel 2016, p. 86-87) : on trouve le premier (piano dans l'aigu) lorsque Wayne et Alfred évoquent Rachel (16:28-17:13) et le second (violoncelle) lorsque Wayne évoque la mort de ses parents à Gordon (02:29:34).

Le quasi mono-thématisme de la partition d'Elfman ne permettant pas des jeux de combinaisons thématiques pour structurer son discours, c'est une dialectique fondée sur les différenciations de langages musicaux plutôt que thématiques qui est retenue par

11 Dans The Dark Knight, Vasco Hexel l'associe d'abord à Gordon - qu'un temps l'on qu'on croit mort - puis à Rachel qui, elle, meurt bel et bien. Les deux motifs sont rassemblés dans The Dark Knight à 01:33:15, quand Rachel vient de mourir et que sa voix, en off, lit sa lettre de manière posthume à Alfred. 
le compositeur ${ }^{12}$. De son côté, Zimmer a la possibilité de recourir aux leitmotive, avec des oppositions ou des superpositions thématiques, voire des citations en l'absence à l'écran du protagoniste évoqué par la musique. Sur ce dernier point, l'exemple le plus spectaculaire intervient au moment où Batman, aux commandes de sa "Bat' " (engin volant en forme de chauve-souris), emporte la bombe (02:29:51-02:30:10) alors que résonne le motif rythmique de son ennemi Bane signifiant que, même mort, ce dernier peut encore nuire mortellement à Gotham. À partir de 02:30:11, la tierce de Batman et le rythme de Bane se superposent. À 02:30:26, enfin, la rythmique binaire de Batman s'impose : le héros a réussi sa mission et sait, à ce moment-là, qu'il aura non seulement la vie sauve - c'est l'autopilote qu'il a lui-même programmé qui va conduire la "Bat' » et emmener la bombe loin de Gotham -, mais qu'il va surtout offrir à sa ville l'exemple de son sacrifice dont il espère qu'il inspirera la population.

\section{MUSIQUE ET SOUND DESIGN : ENTRE RÉALISME ET LÉGENDAIRE}

Dans les scènes d'action du Batman de Burton, c'est essentiellement à la musique que l'on doit le dynamisme de l'ensemble : mixée à fort volume, elle souligne les changements de plan et les détails de l'action. Lors des rares pauses musicales, on peut entendre distinctement certains bruits - train et monnaie (plans 12-14) et pas de Batman (plan 16) dans la première scène (figure 2) - tandis qu'ils sont relégués au second plan, voire absents à d'autres moments où ils sont pris en charge par la musique avec du mickeymousing ${ }^{13}$. S'il s'agit, dans le premier Batman, plutôt de superposition entre bruit et musique (le swoosh du plan 20, figure 2), la bande-son de The Dark Knight Rises atteste d'une fusion bruit/musique au sein de laquelle la frontière entre ces deux composantes sonores devient indiscernable ${ }^{14}$. Vasco Hexel propose une cartographie de la zone de croisements entre musique et sound design (exemple 11), avec l'ensemble des paramètres concernés, pouvant indifféremment ressortir de la musique ou du sound design :

12 "As the leitmotivic approach is effectively impossible, Elfman uses different techniques to achieve a comparable result. In particular, he uses triple time in contrast to duple time, major key in contrast to minor key, and the whole-tone scale in opposition to the romantic idiom, these musical ideas being used to create and underline the sense of duality both within Bruce-Batman's character and in his relationship with the joker» (Halfyard 2004, p. 62).

13 On parle de mickeymousing en référence aux dessins animés de la célèbre souris de Walt Disney pour caractériser les moments où des instruments proposent, de manière exactement synchrone, un équivalent musical des actions et des mouvements à l'image.

14 On peut rapprocher cette conception sonore globale de la bande-son de la musique concrète - dont on peut d'ailleurs faire remonter les premiers exemples aux débuts du cinéma sonore (Langlois 2012). 


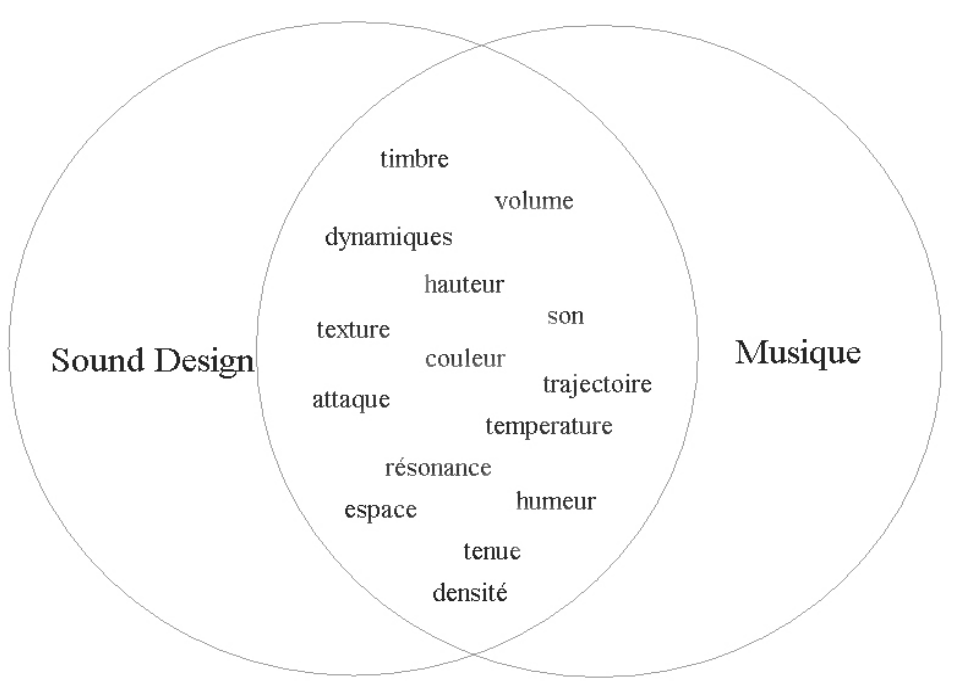

Exemple 11 : Territoire sonore partagé et frontière conceptuelle entre sound design et musique (Hexel 2017, p. 114), tous droits réservés. Schéma reproduit et traduit avec l'aimable autorisation de Rowman \& Littefield.

\section{Conditions techniques de la "narration sonore"}

Connu pour sa maîtrise technique et son souci de la perfection, Nolan travaille la «narration sonore ", soit la capacité du son à prendre en charge l'histoire ${ }^{15}$ - une conception rendue désormais possible par l'évolution technologique de ces vingt dernières années avec l'arrivée de la technologie numérique (Kerins 2015, p. 133-155). Le logiciel historiquement pionnier du mixage sonore numérique - et toujours leader à l'heure actuelle dans sa catégorie ${ }^{16}$-, Pro Tools, a permis d'augmenter considérablement le nombre de pistes au mixage sans ajout de bruit de fond ni perte de qualité (256 pistes qui peuvent être encore doublées en 512) et la facilité d'utilisation permet de multiples expérimentations favorisant la créativité de l'équipe chargée de la postproduction sonore. Par rapport aux films antérieurs au virage numérique, les systèmes de codages audiophoniques numériques permettent aussi de mieux situer les sons dans l'espace, d'éclaircir les paroles, de donner un plus grand réalisme aux ambiances et de restituer plus fidèlement la musique. En plus des systèmes multicanaux traditionnels, The Dark Knight Rises a été conçu pour 1'IMAX sound ${ }^{17}$.

15 Selon Zimmer, "Chris is only interested in me understanding the narrative; the thing which we're most interested in is that I get to be this sort of parallel voice " (Zimmer, cite dans Hyman 2012).

16 Hans Zimmer utilise essentiellement les logiciels Pro Tools (développeur : Avid) et Cubase (développeur : Steinberg).

17 L'IMAX sound accompagne le gigantisme de l'écran imax (pour « Image Maximum », soit un écran de 24 sur 32 mètres) avec une puissance de 14000 watts diffusés par six canaux sonores (quatre derrière l'écran, deux sur les côtés) et une bande de fréquences à la fois très large et d'une très grande finesse dans les aigus et les graves. 
Si dans le premier Batman les sons sont essentiellement constitués des prises de son directes renforcées, ils sont pour la plupart totalement recréés par le sound designer dans The Dark Knight Rises, formant des associations uniques au service de la narration. Dans une vidéo consacrée au sound design du film, le superviseur des effets sonores, Richard King, évoque les cinq catégories de sons qui composent le son de la «Bat » : Jet, Blade, Growl, Buzz, Flanger et Moan. Se trouvent ainsi mêlés des sons réalistes (réacteur d'avion), des sons qui partagent une vague analogie avec l'objet (hélice et lame de la tondeuse à gazon), des sons provenant de processeurs d'effets (buzz et flanger), des sons qui partagent l'imagerie visuelle du film (cri de la chauve-souris) et des sons d'ordre tout à fait psychologique, connotant la violence bestiale, tels que les grognements de chien (growl). La dissociation de la personnalité Wayne/Batman n'étant pas prise en charge musicalement par Zimmer ${ }^{18}$, elle s'exprime au niveau du timbre de la voix et de son traitement ; la voix de Wayne est ainsi monophonique, issue du direct ou de la perche, tandis que celle de Batman, outre son émission gutturale, est entièrement réenregistrée en post-synchronisation avec une prise de son LCRs (Left/Center/Right/ Surround) donnant l'impression d'une voix qui entoure le spectateur, renforçant sa puissance.

Dans le Batman de Burton, les voix, les bruits et les musiques ne sont réunis qu'au moment du mixage. D'un point de vue professionnel, ce processus de travail correspondait à une division des tâches où chaque technicien réalisait sa partie indépendamment des autres. S'inscrivant dans une approche collective du phénomène sonore, Hans Zimmer parle de sa collaboration rapprochée avec King, mais aussi avec Mel Wesson, le sound designer des ambiances, qui travaille essentiellement avec le logiciel Metasynth :

Richard and I worked really closely together; there is another person there, Mel Welson who came up with his original batflap sounds. [...] Richard, Mel and I were forever crossing the lines between what's sound effect and what's music (Zimmer, cité dans Anonyme 2012)

Zimmer revient en particulier sur sa collaboration avec Mel Wesson :

I've spent longer hours on these more organic Batman scores, worrying about tiny little details, making new sounds and palettes, and building instruments. We've got an armada of technicians working with us. I was forever trying to convince Chris that I wanted it broad but not loud. I wanted it murky and not bright. It had to seem to come from within the movie as opposed to being a score that sits on top of the movie. [...] I brought in Mel Wesson to become a bridge between sound effects and music. He does a lot of the ambient sound design, and you can't quite put your finger on if it's a sound effect or if it's music. He's painting with sound; that's really what he's doing. It was important to create a whole sonic world for these characters to inhabit (ibid.).

18 Cette conception d'une unité une et indivisible du personnage de Wayne/Batman est défendue par Halfyard : "His quest to define himself as more than a child who watched his parents die and to empower himself as a figure of justice rather than only of revenge ultimately leads him to become Batman, and Bruce Wayne's money becomes the means to Batman's ends » (Halfyard 2013, p. 187). 
Le bruit comme point de départ ou d'aboutissement de la musique

Les drones d'atmosphère sont des moments privilégiés pour faire naître une musique qui s'installe par un fondu enchaîné sonore. À 01:45:58, dans un quasi silence, un tank au service de Bane effectue sa ronde (extrait vidéo 1) ; la texture doucement craqueleuse que font ses roues qui écrasent la neige sert d'incipit à une nappe synthétique qui s'affirme progressivement : ré aigu, puis la tierce ré-fa dans le medium-aigu.

Extrait vidéo 1 : Christopher Nolan, The Dark Knight Rises, passage d'un tank, 01:45:5401:58:01 (c) Warner Home Video.

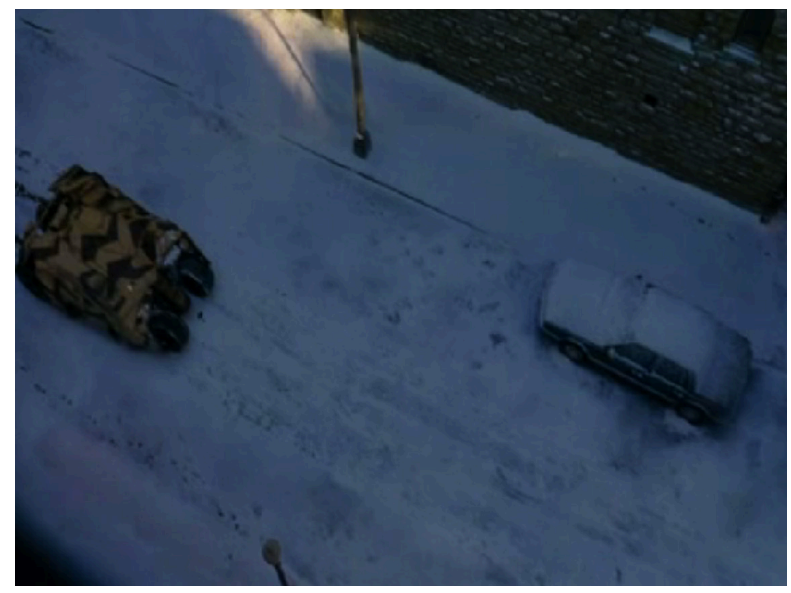

Emblématiques de Batman, les battements d'ailes de la chauve-souris sont présents dans les deux Batman, mais leur utilisation est fondamentalement différente. Dans le premier Batman, il s'agit d'un bruitage réaliste qui reste détaché de la partie musicale à laquelle il ne peut que se superposer (plan 26, figure 2). Dans The Dark Knight Rises, il fait l'objet d'une véritable recherche sonore, au point de devenir un " motif sonore" associé à Batman, le «batflap» («battement d'ailes de chauve-souris », extrait audio 1), élaboré par Wesson dès Batman Begins sur la suggestion de Zimmer : "I suggested Mel go over to the cutting room, to see what sort of thing we needed to start off with. My feeling was that it didn't even really have to be a piece of music. It just had to be something really iconic, like the flapping of ginormous wings or something so " (Zimmer, cité dans Hexel 2016, p. 67).

Il s'agit d'un signal sonore d'ordre plutôt conceptuel, car Batman, en réalité, plane plutôt qu'il ne vole ; toutefois, les récurrences particulières avec la "Bat' » à Wayne renforce cette association avec le vol. Après le générique de début, le "batflap » réapparaît précisément au moment où Fox (Morgan Freeman) présente la " Bat' " (38:49), puis couvre pratiquement toute la séance où la jambe de Wayne est soignée par Alfred pour qu'il puisse, à nouveau, "voler de ses propres ailes "; à la toute fin du film, le signal revient quand Batman est aux commandes de sa « Bat' » (02:29:44). Ce motif sonore identifiant signale la présence du super-héros même en son absence visuelle, par exemple lorsque Gordon ramasse une mèche de feu d'artifice (02:06:36), peu avant que la tierce de Batman (sur l'enchaînement harmonique de la réussite) ne se fasse entendre, alors qu'une chauve-souris de feu s'est déployée dans le ciel (02:07:12).

Extrait audio 7 : Hans Zimmer et Mel Wesson, The Dark Knight Rises, cue "Main Theme ", rythmique à partir des batflaps. Bande originale, 00:00-00:08. Écouter. 
Si la musique naît du bruit, elle peut aussi s'y résorber, le bruit en constituant alors l'aboutissement. Lors de la première tentative de Wayne pour sortir du puits, la musique, après avoir soutenu en "effects underscoring" (voir infra) le chœur "Deschi Basara ", aboutit au bruit brutal et résonnant de la chute de Wayne se cognant contre la paroi du puits (01:48:17), véritable climax sonore qui coupe la musique (extrait vidéo 2); un drone sombre et caverneux assure par la suite la continuité sonore.

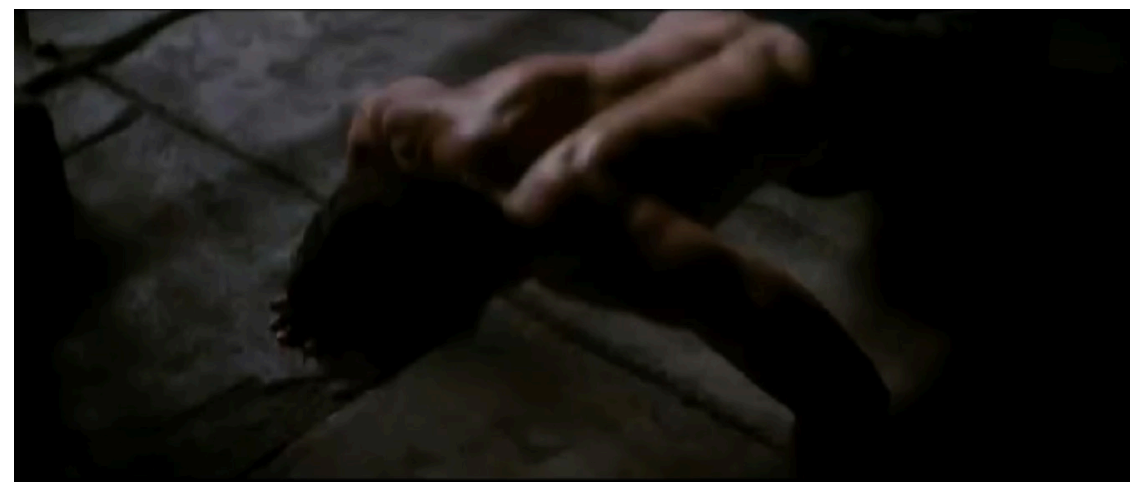

Extrait vidéo 2 : Christopher Nolan, The Dark Knight Rises, première tentative de Wayne pour s'extraire du puits, 01:47:15-01:48:21@ Warner Home Video.

\section{"Effects underscoring"}

À plusieurs reprises, il nous semble assister à une inversion de la hiérarchie traditionnelle entre bruits (en anglais : effects ${ }^{19}$ ) et musique (Sergi 2006), la seconde mettant en valeur les premiers par un procédé que nous qualifierions d' "effects underscoring 20 ", soit une écriture par laquelle la musique se voit destinée à former un écrin émotionnel non plus aux voix, mais aux bruits lors de séquences où ce sont ces mêmes bruits qui, par leur propriétés intrinsèques (organisation rythmique, caractéristiques timbrales), concentrent la signification dramaturgique d'un passage.

À 01:21:04, la visite de Bane dans la Batcave déclenche l'ostinato rythmique du personnage sur une note tenue au violon ; ces éléments invariants s'opposent à la diversité des bruits de clés et de dévisseuses électriques qui, elles, nous renseignent par leur fourmillement sonore sur l'ampleur de l'arsenal militaire du département recherche de Wayne Enterprises, désormais aux mains de Bane. On ne peut toutefois véritablement parler d'effects underscoring ici en ce que la musique, si elle sert bien d'accompagnement aux bruits, ne montre pas d'évolution manifeste - on parlera plutôt d'un drone d'accompagnement.

\footnotetext{
19 Terme abrégé pour "sound effects " qui comprend à le choix des bruits réalistes et des ambiances (provenant de sons directs, d'un bruiteur, de banques de sons), ainsi que la création de sons originaux (sound design).

20 L'underscoring désigne traditionnellement un type particulier d'écriture musicale pour l'écran destinée à accompagner émotionnellement les dialogues, tout en s'adaptant à la tessiture des voix et à leur débit.
} 
Extrait vidéo 3 : Christopher Nolan, The Dark Knight Rises, visite de Bane dans la Batcave, 01:21:04-01:21:14 (C) Warner Home Video.

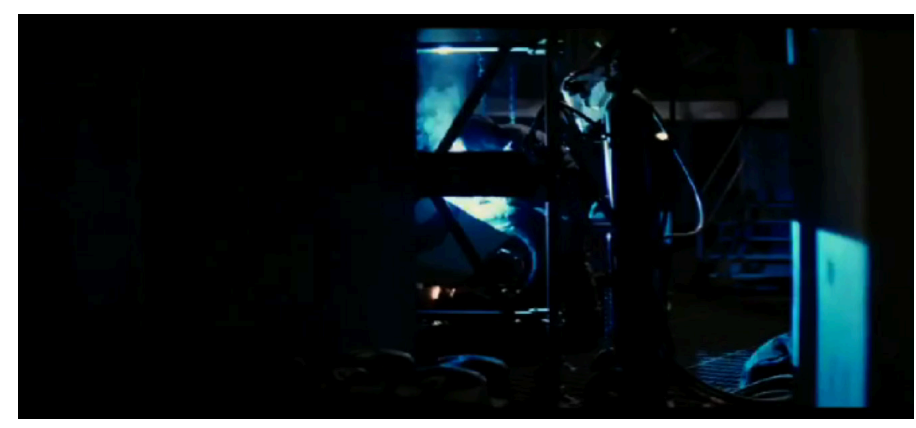

Il en va différemment dans les deux actions les plus dramatiques et les plus spectaculaires du film liées à Bane : la prise d'otages à la bourse et l'explosion des égouts, deux séquences accompagnées d'un bout à l'autre par une nappe électroacoustique tenue sur un accord de ré mineur dans le registre grave. Dans les deux cas, les dialogues sont rares - inexistants dans le second - et c'est à une dramaturgie presque entièrement portée par les bruits que s'en remet Nolan. La prise d'otages repose sur quatre ambiances - foule (bruyante ou paniquée), tirs, sirènes de police et absence de bruit (notée " $\mathrm{x}$ ", voir figure 1) - et sur la présence musicale de discrètes strates en ostinato de cordes qui viennent, à trois reprises, se superposer à la nappe synthétique en ré mineur ; ces éléments sont répartis en une structure que l'on pourrait assimiler à une forme musicale à " refrains " ${ }^{21}$ (soit une « forme rondo » dans la typologie des formes musicales traditionnelles). Les moments non bruités, seulement accompagnés de musique, confèrent à Bane, par contraste avec ce qui précède ou succède, des qualités de maîtrise de soi et de contrôle des événements. Son " ostinato identifiant " rythmique revient à la fin de la séquence, alors qu'il a réussi à s'échapper de la bourse en s'emparant d'un otage. Avant cet ostinato, la musique ne possède aucun rôle thématique, mais elle n'est pas non plus statique à la manière d'un drone puisque des ostinatos reviennent périodiquement ; elle se trouve précisément en situation d'effects underscoring pour communiquer une sensation de tension et d'urgence (les ostinatos de cordes sont vraisemblablement insérés grâce à la technique du stem scoring - voir plus loin).

\begin{tabular}{|l|l|l|l|l|l|l|l|l|l|l|}
\hline $41: 52$ & $42: 20$ & $42: 31$ & $43: 12$ & $43: 58$ & $44: 03$ & $44: 27$ & $44: 37$ & $44: 40$ & $44: 45$ & $45: 08$ \\
\hline foule & tirs & $\mathrm{x}$ & sirènes & $\mathrm{x}$ & sirènes & $\mathrm{x}$ & tirs & foule & $\mathrm{x}$ & foule \\
\hline $\begin{array}{l}\text { Nappe } \\
\text { ré min. }\end{array}$ \\
rest. cordes cordes \\
\hline
\end{tabular}

Figure 1: Christopher Nolan, The Dark Knight Rises, structure sonore "en rondo" de la prise d'otage de la bourse, 41:52-45:15.

Lorsque les rues de Gotham explosent à cause des explosifs contenus dans le béton des canalisations enfoui par les hommes de Bane (01:29:39-01:30:50) -, la même nappe synthétique en ré mineur revient, seulement variée, cette fois, par un

21 Ces refrains correspondant à l'absence de bruit ; notons seulement à 42:40 les bruits de respiration de Bane dans son masque, qui font paradoxalement ressortir le silence dont il est entouré - marque de la crainte qu'il inspire. 
lent sforzando aux cuivres : en fonction des plans - plan aérien de la ville, stade, salle des officiels qui assiste au match derrière une vitre, voiture de Blake, égouts dans lesquels sont coincés les policiers - les bruits d'explosions varient, eux, constamment de volume et de sonorité. L'effect underscoring consiste ici en la conception d'une lente gradation musicale qui dramatise les explosions et les oriente temporellement ${ }^{22}$.

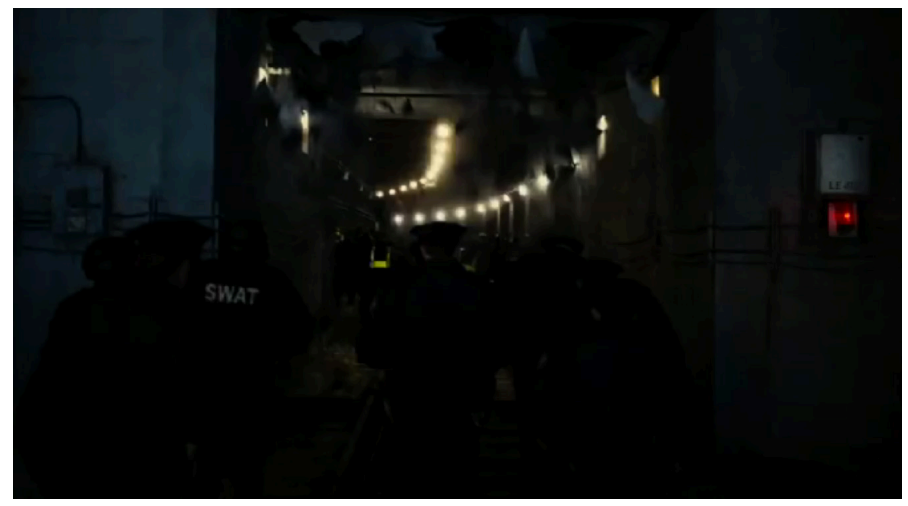

Extrait vidéo 4: Christopher Nolan, The Dark Knight Rises, explosion des canalisations de Gotham, 01:29:39-01:30:50 (C) Warner Home Video.

Plus loin (01:47:15, extrait vidéo 2), lors de la première tentative d'extraction du puits de Wayne, Zimmer installe une séquence de quatre accords répétés (Dm, $\mathrm{Dm} / \mathrm{E}, \mathrm{Dm} / \mathrm{F}, \mathrm{C}$ ) jouée aux cordes graves qui prend peu à peu de l'ampleur, avec quelques notes traînantes de cuivres et un contrechant (toujours aux cordes) lors de la troisième récurrence. Ce matériau musical minimal, aux sonorités soigneusement choisies, accompagne la déclamation diégétique «Deschi Basara », plus «bruit » que " chant ", scandée par les prisonniers de Bane qui encouragent Wayne. Ce sont les traitements sonores de ce chœur - augmentation du nombre de voix et du volume, traitements acoustiques variant rapidement en fonction du positionnement de la caméra - qui assurent la progression dramatique de la séquence jusqu'au climax de la chute, tandis que la musique, fonctionnant par boucle de quatre accords, accentue en effects underscoring l'effet répétitif et lancinant de cette déclamation.

\section{Manipulations des bruits diégétiques}

L'omniprésence et la finesse du travail sur les bruits dans The Dark Knight Rises de Nolan vont dans le sens de la "réalité augmentée » souhaitée par le réalisateur ${ }^{23}$. Lorsqu'à l'évidence des bruits sont omis, ou exagérément sous-mixés, c'est tout le conditionnement du spectateur qui se trouve interrogé ; cette déstabilisation produit une déréalisation qui semble pouvoir être ainsi interprétée : " ce qui va arriver est si grave qu'on pourrait douter de sa réalité ». Du réalisme, on bascule alors dans le

22 Michel Chion évoque la capacité de la musique à créer des «lignes de fuite temporelle » par crescendo (Chion 1995, p. 209) vers leur aboutissement : ici, le chaos.

23 Dans un entretien avec Geoff Boucher (Boucher 2008), Christopher Nolan confie rechercher une réalité augmentée ("heightened reality») privilégiant les décors réels et limitant la part de l'imagerie informatique. 
légendaire, la musique prenant cette fois le relais des bruits pour exprimer l'aspect mythique d'un moment dont l'ampleur laisse supposer qu'il laissera une trace dans l'histoire des hommes. Trois séquences sont particulièrement éloquentes à cet égard.

À partir du moment où Blake révèle le plan machiavélique de Bane - l'addition d'explosif dans le béton des canalisations de Gotham -, la ville s'apprête à basculer dans l'horreur, alors même que ses habitants sont rassemblés dans le stade. Pour souligner ce moment pivot, tous les bruits diégétiques sont coupés pour laisser entendre un jeune garçon chanter l'hymne américain (01:27:44): les couleurs jaunes des gradins et des supporters, le beau temps et la voix très pure du jeune garçon, avec une réverbération généreuse, entrent directement en contraste avec le drame qui couve. Aucun bruit n'accompagne Bane qui s'approche du stade, ni les milliers de supporters, ni les policiers qui courent dans les égouts pour s'enfuir. On n'entend plus que les voix : celle de de Blake filtrée par le téléphone qui avertit Foley (Matthew Modine) du piège qui va se refermer sur les policiers, celle de Foley qui donne l'ordre à ses hommes de se replier, enfin celle de Bane momentanément touché par la beauté $\mathrm{du}$ chant. Lorsque le jeune garçon termine l'hymne, les sons diégétiques reviennent par les applaudissements des spectateurs; Gotham va alors sombrer dans le chaos.

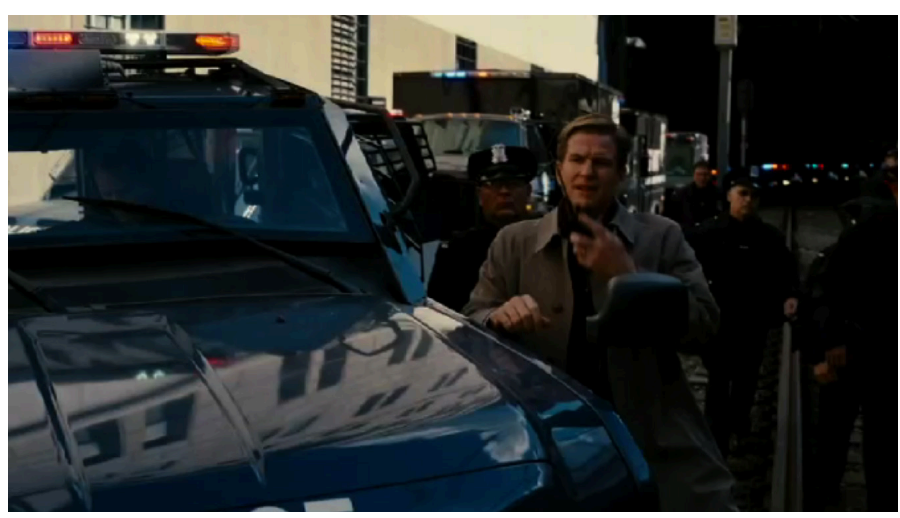

Extrait vidéo 5 : Christopher Nolan, The Dark Knight Rises, hymne américain chanté dans le stade, 01:27:36-01:29:00 (C) Warner Home Video.

Lorsque Miranda, son poignard plongé dans les entrailles de Batman, raconte à celui-ci (02:17:47) la "légende du mercenaire ", tous les bruits extérieurs cessent, et les bruits diégétiques de cette légende sont eux-mêmes très assourdis au bénéfice du " thème légendaire " de Bane (exemple 8) - que l'on aperçoit un court moment sans son masque. C'est finalement l'action parallèle - Gordon qui trouve le camion qui porte la bombe - qui déclenche le retour des bruits

$\mathrm{Au}$ moment où la "Bat " s'élève, emportant la bombe menaçante en son sein, les bruits disparaissent complétement - y compris celui, d'ordinaire tonitruant, du moteur de la "Bat ». Le thème du deuil est cité au violoncelle solo, alors que Gordon a découvert la véritable identité de Batman ; à ce moment, tout le monde, y compris le spectateur, est persuadé que le super-héros va se sacrifier pour sa ville, et c'est cet aspect légendaire que prend en charge la disparition des bruits diégétiques au profit de la musique. Le bruit de la bombe qui touche le sol, avant d'être tractée par « la Bat' ", provoque le retour des bruits... qui disparaissent une nouvelle fois (02:30:52) pour laisser retentir le " thème psychanalytique » de Batman alors que le super-héros 
apparaît en gros plan. Ces jeux sur les bruits diégétiques, dans un environnement bruité extrêmement réaliste, créent un espace symbolique très puissant où se joue l'aspect légendaire des personnages.

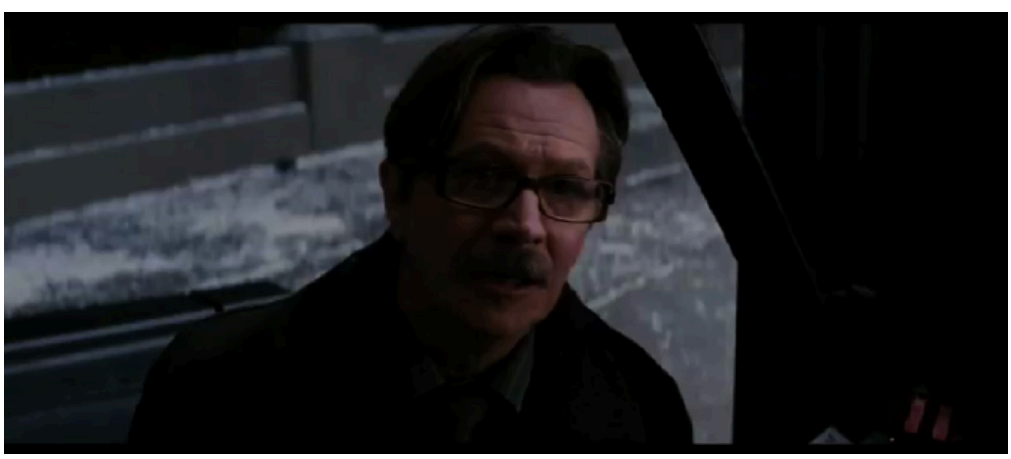

Extrait vidéo 6: Christopher Nolan, The Dark Knight Rises, décollage final de "la Bat'», 02:29:15-01:29:51 (c) Warner Home Video.

Utilisation du bruit comme point d'entrée ou point de sortie de la musique, "effects underscoring ", manipulations des bruits diégétiques au bénéfice de la musique, constituent les principales stratégies pour créer un continuum entre bruit et musique, une zone frontière perméable servant de pivot entre le réalisme et le légendaire, en un équilibre sans cesse réajusté. Le traitement extrêmement minutieux des bruits, au service d'une mise-en-scène très réaliste, s'avère selon nous conditionner l'approche musicale de Zimmer - raison pour laquelle nous avons souhaité d'abord présenter le travail sur les bruits avant d'en venir aux stratégies compositionnelles de Zimmer que nous allons à présent comparer à celles d'Elfman dans plusieurs scènes d'action.

RAPPORTS RYTHMIQUES À L'IMAGE DANS LES SCĖNES D'ACTION : SYNCHRONISMES ET OSTINATOS

Si le thème super-héroïque est un ingrédient constitutif des films de super-héros, la scène d'action est l'autre marqueur emblématique du genre. C'est en effet dans l'action que se révèle le personnage super-héroïque : Clark Kent y devient Superman, Peter Parker, Spiderman ou Bruce Wayne, Batman. En-dehors de ces moments d'action, rien, finalement, ne distingue plus le super-héros d'un individu ordinaire, indistinction généralement suivie par la musique. Nous proposons d'étudier deux aspects rythmiques des musiques de scène d'action : les synchronismes et le maniement des ostinatos.

\section{Synchronismes}

L'écriture d'Elfman est attentive à capter et à souligner tous les détails de l'image, tant les mouvements des personnages à l'écran que les changements de plans, comme nous le montre une analyse de la première séquence (figure 2) ; Batman y châtie deux malfrats qui s'en prenaient à un père, sa femme et leur garçon ${ }^{24}$. C'est l'occasion,

24 Cette scène fait écho à la propre histoire de Batman qui a vu ses parents mourir sous ses yeux dans les mêmes conditions. 
dès le début du film, de présenter le super-héros en action. La musique épouse la structure visuelle avec des changements de dispositifs instrumentaux calés sur les changements de plan ; seule l'installation d'un ostinato, à partir du plan 17, établit une continuité sonore (pas de séparation dans la colonne "Musique » du plan 17 au plan 21) même si, là encore, plusieurs détails continuent d'être pris en charge par la musique (ils sont représentés entre crochets), y compris par du mickeymousing comme ce glissando de l'orchestre lorsque Batman déploie ses ailes (plan 18) ou le coup de gong au moment où il se redresse (plan 24). Les moments non musicalisés sont très rares : ce sont les bruits de train sur le plan 12 et les pas de Batman sur le plan 16. On relèvera également un passage en underscoring pendant le discours entre les deux malfrats : les cordes graves en pizz. puis des tenues cordes/cuivres dans le grave laissent parfaitement passer les voix, tandis que de courtes interventions viennent ponctuer leur dialogue : deux intervalles mélodiques de tierces majeures viennent détacher la plaisanterie à propos de la carte « American express » (04:44-04:50) et des trémolos aux cordes medium accompagnent l'évocation de la rumeur inquiétante de la chauve-souris (05:08-05:15).

\begin{tabular}{|c|c|c|c|c|}
\hline Temps & $\mathrm{N}^{\circ}$ & Type de plan & Image & Musique \\
\hline $03: 47$ & 1 & moyen & $\begin{array}{l}\text { Entrée de la famille (père, mère } \\
\text { et garçon) dans une ruelle } \\
\text { sombre }\end{array}$ & $\begin{array}{l}\text { motif de trois notes à la harpe, } \\
\text { quelques percussions (castagnettes) et des } \\
\text { notes de basson }\end{array}$ \\
\hline 03:56 & 2 & plongée ensemble & $\begin{array}{l}\text { La famille prend un tournant } \\
\text { dans la ruelle. }\end{array}$ & $\begin{array}{l}\text { notes tenues de cordes dans le registre médium- } \\
\text { notes de basson }\end{array}$ \\
\hline 04:00 & 3 & moyen & \multirow[t]{2}{*}{ Un malfrat demande de l'argent } & motif arpégé ascendant (la do mi fä) \\
\hline 04:07 & 4 & moyen & & notes de basson \\
\hline $04: 10$ & 5 & rapproché taille & Commentaire du malfrat & réitération du motif arpégé ascendant \\
\hline $04: 15$ & 6 & rapproché taille & Le père se fait assommer & oscillation dramatique en crescendo \\
\hline 04:18 & 7 & rapproché taille & $\begin{array}{l}\text { Le deuxième malfrat menace } \\
\text { la mère et son garçon }\end{array}$ & \multirow{4}{*}{$\begin{array}{l}\text { enclenchement d'un ostinato en croches } \\
\text { aux bois, à la harpe et aux cordes en pizz., } \\
\text { auxquels se joignent des timbales en } p p \\
\text { léger crescendo sur la fin }\end{array}$} \\
\hline 04:20 & 8 & moyen & $\begin{array}{l}\text { Le premier malfrat les rejoint } \\
\text { les deux malfrats s'enfuient }\end{array}$ & \\
\hline $04: 27$ & 9 & rapproché taille & La femme hurle & \\
\hline 04:29 & 10 & plongée ensemble & $\begin{array}{l}\text { La femme et l'enfant se } \\
\text { précipitent sur le corps inerte } \\
\text { du père }\end{array}$ & \\
\hline $04: 31$ & 11 & plongée ensemble & Apparition de Batman & $\begin{array}{l}\text { motif de Batman à la trompette, } \\
\text { sur cordes graves et gong }\end{array}$ \\
\hline 04:39 & 12 & plongée moyen & \multirow{3}{*}{$\begin{array}{l}\text { Les deux malfrats discutent } \\
\text { et évoquent la chauve-souris }\end{array}$} & \multirow{3}{*}{$\begin{array}{l}\text { courte pause musicale (bruits réalistes : train, } \\
\text { sirène, monnaie), puis underscoring avec une } \\
\text { atmosphère bruitiste composée de } \\
\text { pizz., de contrebasses, de notes tenues aux } \\
\text { cordes graves, de trémolos de cordes et } \\
\text { sonorités hexatoniques au vibraphone }\end{array}$} \\
\hline $04: 50$ & 13 & moyen & & \\
\hline 05:03 & 14 & $\begin{array}{l}\text { série de trois gros } \\
\text { plans en champ/ } \\
\text { contrechamp }\end{array}$ & & \\
\hline 05:17 & 15 & demi-ensemble & $\begin{array}{l}\text { Les deux malfrats discutent, } \\
\text { apparition de Batman } \\
\text { au second plan }\end{array}$ & $\begin{array}{l}\text { répétition dans des registres de plus en } \\
\text { plus aigus de l'incipit du motif de } \\
\text { Batman }\end{array}$ \\
\hline $05: 33$ & 16 & moyen & Poursuite de la discussion & $\begin{array}{l}\text { motif de Batman joué lentement en notes } \\
\text { tenus aux cordes graves, puis courte pause } \\
\text { musicale où l'on entend les pas de Batman }\end{array}$ \\
\hline 05:49 & 17 & plongée moyen & $\begin{array}{l}\text { Vue subjective de Batman } \\
\text { sur les malfrats }\end{array}$ & \multirow{3}{*}{$\begin{array}{l}\text { mise en place d'un nouvel ostinato en } \\
\text { croches percussions cordes, bois et piano } \\
\text { [glissando d'orchestre] }\end{array}$} \\
\hline $05: 51$ & 18 & $\begin{array}{l}\text { contre-plongée } \\
\text { moyen }\end{array}$ & Batman étend ses ailes & \\
\hline 05:52 & 19 & plongée moyen & $\begin{array}{l}\text { Vue subjective de Batman } \\
\text { sur les malfrats qui s'enfuient }\end{array}$ & \\
\hline $05: 54$ & 20 & $\begin{array}{l}\text { contre-plongée } \\
\text { moyen }\end{array}$ & Batman saute & \multirow[t]{2}{*}[\text{swoosh}]{} \\
\hline 05:56 & 21 & $\begin{array}{l}\text { Série de trois } \\
\text { champ/contrechamp }\end{array}$ & $\begin{array}{l}\text { Les malfrats tirent sur Batman } \\
\text { qui tombe à terre }\end{array}$ & \\
\hline 05:59 & 22 & moyen & Batman est à terre & interruption de l'ostinato, note tenue \\
\hline 06:01 & 23 & rapproché taille & Les malfrats veulent s'enfuir & \multirow{5}{*}{$\begin{array}{l}\text { reprise de l'ostinato } \\
\text { [son de gong et « déchaînement » } \\
\text { musical en mickeymousing sur le } \\
\text { déploiement d'ailes] }\end{array}$} \\
\hline 06:05 & 24 & gros plan de dos & $\begin{array}{l}\text { Batman se redresse et déploie } \\
\text { ses ailes }\end{array}$ & \\
\hline 06:08 & 25 & deux gros plans & $\begin{array}{l}\text { Regards apeurés des deux } \\
\text { malfrats }\end{array}$ & \\
\hline 06:10 & 26 & Rapproché taille & $\begin{array}{l}\text { Batman replie ses ailes et fait } \\
\text { face aux deux malfrats ; } \\
\text { la bataille s'engage }\end{array}$ & \\
\hline 06:11 & 27 & $\begin{array}{l}17 \text { plans rapprochés } \\
\text { gros plans }\end{array}$ & $\begin{array}{l}\text { Bagarre (le premier malfrat est } \\
\text { occis par un coup de pied) }\end{array}$ & \\
\hline 06:34 & 28 & ensemble plongée & $\begin{array}{l}\text { Pieds du malfrat suspendus } \\
\text { dans le vide }\end{array}$ & \multirow[t]{2}{*}{$\begin{array}{l}\text { arrêt de l'ostinato, motif de Batman } \\
\text { en notes longues aux cordes graves }\end{array}$} \\
\hline $06: 37$ & 29 & $\begin{array}{l}2 \text { plans champ/ } \\
\text { contrechamp }\end{array}$ & $\begin{array}{l}\text { Batman tient le malfrat en } \\
\text { respect; le malfrat lui demande } \\
\text { «Qui es-tu? ? }\end{array}$ & \\
\hline
\end{tabular}




\begin{tabular}{|c|c|c|c|c|}
\hline $06: 40$ & 30 & gros plan & Batman : « Je suis Batman. » & \multirow{6}{*}{$\begin{array}{l}\text { coups de tambours qui relancent la } \\
\text { citation du motif de Batman aux cuivres }\end{array}$} \\
\hline $06: 42$ & 31 & moyen & Batman jette le malfrat à terre & \\
\hline $06: 44$ & 32 & rapproché & Le malfrat gît à terre & \\
\hline $06: 47$ & 33 & moyen & Batman saute dans le vide & \\
\hline $06: 49$ & 34 & rapproché & Le malfrat se relève & \\
\hline $06: 53$ & 35 & $\begin{array}{l}\text { Moyen } \\
\text { contre-plongée }\end{array}$ & $\begin{array}{l}\text { Le malfrat se penche pour voir } \\
\text { où est passé Batman }\end{array}$ & \\
\hline $06: 55$ & 36 & Ensemble, plongée & La ruelle, vide & \multirow[t]{2}{*}{ balancement d'accord (Gm/EM) } \\
\hline $06: 58$ & 37 & $\begin{array}{l}\text { Moyen } \\
\text { contre-plongée }\end{array}$ & $\begin{array}{l}\text { Retour sur le malfrat en train } \\
\text { de pleurer }\end{array}$ & \\
\hline
\end{tabular}

Figure 2: Tim Burton, Batman, description analytique de la séquence comprenant l'attaque et la première apparition de Batman, 03:47-07:03 ${ }^{25}$.

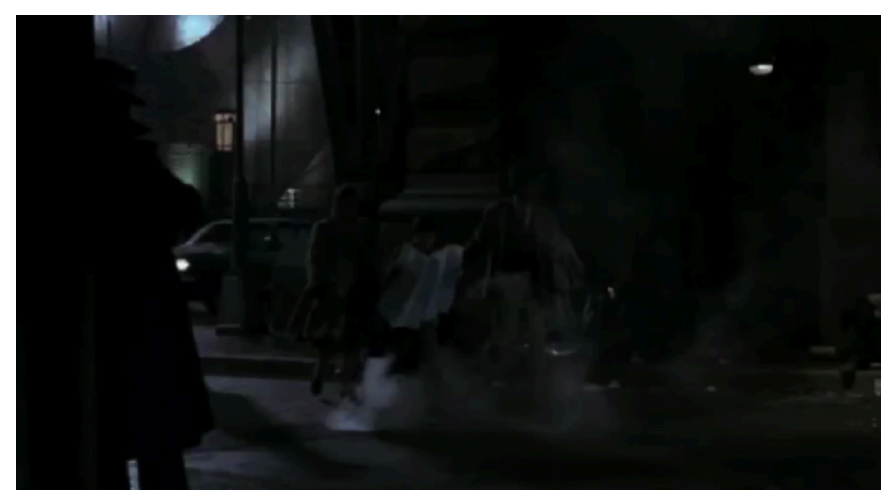

Extrait vidéo 7 : Tim Burton, Batman, attaque et première apparition de Batman, 03:47-07:03 (C) Warner Home Video.

Par comparaison, l'approche musicale chez Zimmer est bien moins attentive aux détails des actions montrées à l'écran, comme nous allons le voir. La séquence d'ouverture étant entièrement centrée sur Bane ${ }^{26}$, nous avons choisi la scène dans laquelle Batman vient secourir Kyle, prise au piège par les hommes de Bane chez Daggett (Ben Mendelsohn) auprès duquel elle venait réclamer le programme « Table rase ». L'absence de séparation dans la colonne "Musique " du plan 7 au plan 22 révèle la longueur d'utilisation d'un même ostinato (déjà présent des plans 1 à 4) sur 43 sec., quand Elfman maintenait au maximum son ostinato pendant 10 sec. (du plan $17 \mathrm{au}$ 21). Seul l'envol de la «Bat' »-quand Batman a repris le contrôle de la situation - provoque un changement d'ostinato (plan 23).

L'observation de la figure 3 montre que les synchronismes de plans sont peu fréquents (en moyenne, un synchronisme tous les quatre plans, quand Elfman marquait tous les changements de plans, y compris des synchronismes avec des mouvements visuels à l'intérieur d'un même plan - plan 15), mais surtout qu'ils ont changé de nature. À la discontinuité elfmanienne (encore ponctuellement utilisée, telle le sfz de cuivres

25 Le swoosh (05:54) a sans doute été rajouté par au mixage car il n'est pas présent sur la bande originale.

26 C'est toutefois la même stratégie qui est adoptée. S'il y a bien quelques points de synchronisme avec des changements de textures (à 04:17 : accents des cordes en cluster sur le plan des partisans de Bane sortant du second avion ; à 06:05 : interruption puis reprise de la rythmique juste au moment où que Bane est hélitreuillé), ni les coups de pistolet, ni les révélations successives du visage de Bane et du second avion, ni le démembrement violent du premier avion ne sont marqués musicalement ; inversement, l'arrivée des tambours à 05:34 ne souligne aucun fait saillant à l'image. 
du plan $6^{27}$ ), Zimmer préfère jouer avec le déclenchement ou l'arrêt des différentes strates sonores, une technique que nous proposons d'appeler "stem scoring ", soit une composition musicale conçue en plusieurs strates que le compositeur ou le mixeur peut à loisir déclencher ou taire en fonction de l'image ${ }^{28}$. Le plan 7 correspond au début de la bagarre avec les hommes de Bane, ce qui déclenche l'arrivée des strates en ostinato de rythmique électroniques et de cordes graves. La courte réplique de Batman à Kyle sur son éthique (" on ne tue pas ») provoque un court underscoring grâce au "stem scoring " : la disparition de la strate en ostinato des cordes graves au bénéfice d'une nouvelle couche, moins dense, aux cuivres dans le grave qui permet de dégager les fréquences vocales de Batman (il a à ce moment sa voix gutturale). L'arrivée en personne de Bane est marquée par l'apparition de la couche vocale «Deschi Basara » (plan 20), avant que la rythmique binaire de Batman ne remplace l'ostinato de Bane, une fois que le héros a repris le contrôle de la situation (à partir du plan 23).

\begin{tabular}{|c|c|c|c|c|}
\hline Temps & $\mathrm{N}^{\circ}$ & Plans & Description & Musique \\
\hline 53:02 & 1 & Ensemble & Bane et ses hommes arrivent & \multirow{4}{*}{$\begin{array}{l}\text { motif de Bane en ostinato aux tambours } \\
\text { (sonorité sourde) sur une texture en } \\
\text { glissandi aux cordes dans le suraigu }\end{array}$} \\
\hline 53:05 & 2 & Rapproché taille & Kyle se redresse & \\
\hline 53:06 & 3 & Caméra épaule & Bane et ses hommes s'approchent & \\
\hline 53:10 & 4 & Moyen & $\begin{array}{l}\text { Kyle prend Daggett en otage, les ho } \\
\text { continuent de s'approcher }\end{array}$ & \\
\hline 53:16 & 5 & $\begin{array}{l}\text { Contre-plongée, } \\
\text { ensemble }\end{array}$ & Batman fait son apparition & silence \\
\hline 53:18 & 6 & Caméra épaule & Kyle menace toujours Daggett & [sfz de cuivres] \\
\hline $53: 20$ & 7 & Caméra épaule & Kyle donne son premier coup de pied & \multirow{16}{*}{$\begin{array}{l}\text { reprise de l'ostinato avec ajout de deux } \\
\text { strates (rythm. électro, tambours brillants) } \\
\text { [Bruissement de cape] } \\
\text { ajout d'une strate (cordes graves) } \\
\text { suppression de cette strate (cordes graves) } \\
\text { et ajout d'une strate mélodique: tierce de } \\
\text { Batman jouée deux fois sur les } \\
\text { progressions harmoniques : « attente» } \\
\text { puis « danger » } \\
\text { ajout d'une strate } \\
\text { (notes piquées de trompette) }\end{array}$} \\
\hline $53: 21$ & 8 & Demi-ensemble & Saut de Batman & \\
\hline $53: 22$ & 9 & Moyen & Atterrissage de Batman & \\
\hline $53: 23$ & 10 & Caméra épaule & Bagarre & \\
\hline 53:27 & 11 & Rapproché taille & Visage angoissé de Daggett & \\
\hline 53:28 & 12 & Caméra épaule & Bagarre & \\
\hline $53: 37$ & 13 & Rapproché taille & $\begin{array}{l}\text { Batman interdit à Kyle } \\
\text { l'emploi des armes }\end{array}$ & \\
\hline $53: 40$ & 14 & $\begin{array}{l}3 \text { plans, Caméra } \\
\text { épaule }\end{array}$ & reprise de la bagarre & \\
\hline $53: 44$ & 15 & Ensemble & $\begin{array}{l}\text { Des coups de feu éclatent } \\
\text { depuis le bâtiment }\end{array}$ & \\
\hline $53: 46$ & 16 & Ensemble & Batman et Kyle fuient & \\
\hline $53: 48$ & 17 & Moyen & Batman et Kyle gagnent la corniche & \\
\hline 53:51 & 18 & Moyen & Batman saute dans sa « Bat » & \\
\hline $53: 52$ & 19 & Rapproché taille & Kyle se retourne pour voir derrière elle & \\
\hline $53: 53$ & 20 & Moyen & Bane s'approche & \\
\hline 53:54 & 21 & Caméra épaule & Kyle se retourne vers Batman et saute & \\
\hline 53:58 & 22 & $\begin{array}{l}\text { Rapproché poitrine } \\
\text { puis déplacement }\end{array}$ & $\begin{array}{l}\text { Kyle s'adresse à Batman, } \\
\text { qui lui répond }\end{array}$ & \\
\hline 54:03 & 23 & Rapproché & Kyle regarde en l'air & \multirow[t]{5}{*}{ rythmique binaire puis ostinato de Batman } \\
\hline 54:04 & 24 & Moyen & $\begin{array}{l}\text { Bane est de dos et voit } \\
\text { décoller la « Bat» }\end{array}$ & \\
\hline 54:09 & 25 & Rapproché taille & Bane est de face, éclairé par la « Bat» & \\
\hline 54:13 & 26 & Demi-ensemble & La « Bat » manœuvre dans le ciel & \\
\hline $54: 20$ & 27 & Large & Survol de la ville de Gotham & \\
\hline
\end{tabular}

Figure 3 : Hans Zimmer, The Dark Knight Rises, description analytique de la séquence de combat de Selina Kyle et Batman chez Daggett, 53:02-54:25.

27 Ce $s f z$ s'apparent plus à un bruitage de type swoosh qu'à un phénomène musical.

28 Ce type d'écriture se rapproche ce qu'Ennio Morricone (Miceli et Morricone 2013, p. 132) appelle l'« écriture modulaire " (" modular writing ") qui sert à désigner la conception d'une partition en plusieurs strates destinées à être enregistrées séparément ("overdub») pour permettre au compositeur d'élaborer sa propre version. Le "stem scoring " est différent en ce que l'éclatement des strates est conçu dès le départ moins pour satisfaire les désirs expérimentaux du compositeur que pour assurer une souplesse d'utilisation au moment du mixage. 


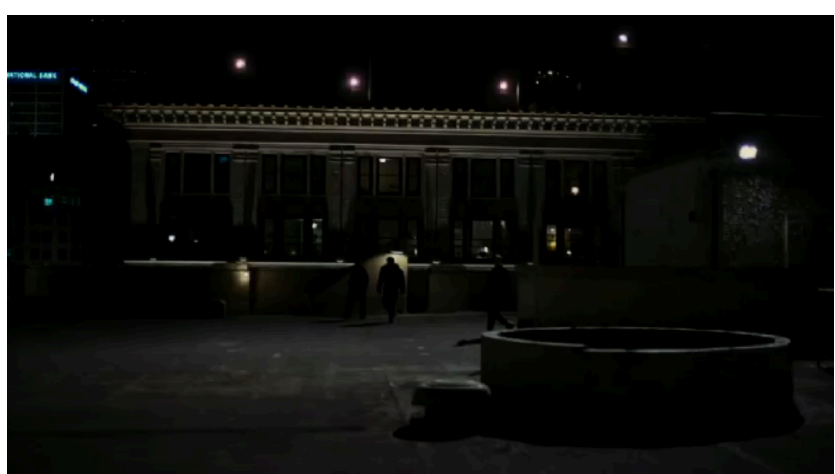

Extrait vidéo 8: Christopher Nolan, The Dark Knight Rises, combat de Selina Kyle et Batman chez Daggett, 53:02-54:21 @ Warner Home Video.

Si le synchronisme reste pratiqué par les deux compositeurs, nous dirons qu'il s'est non seulement inversé proportionnellement, mais surtout qu'il a changé de nature, privilégiant une plus grande continuité chez Zimmer. La plus grande rareté des synchronismes chez ce dernier est révélatrice des nouvelles méthodes de travail liées au numérique, qui impliquent des allers-et-retours constants entre les différents acteurs de la post-production : le film reste jusqu'au bout une forme potentiellement évolutive, toutes ces composantes pouvant être manipulées - il s'agit là d'une évolution majeure du workflow qui peut aussi s'appliquer à la musique ${ }^{29}$. Dans cette perspective où tout est en perpétuel remaniement, une musique dépendante d'un nombre trop élevé de synchronismes serait compliquée à manipuler, au contraire de strates que l'on peut taire ou déclencher en fonction des besoins.

Zimmer reste toutefois attaché à une conception signalétique des thèmes : la musique cite la thématique ou la rythmique associée du protagoniste lorsque celui-ci est présent à l'écran. Tout comme Elfman (plans 15, 16, 28, 30-35), Zimmer donne à entendre les deux éléments du thème du super-héros pendant la scène précédemment étudiée : la tierce mélodique de Batman, énoncée sur la rythmique de Bane (plan 13) lors du dialogue avec Selina Kyle, puis son « ostinato identifiant » lors du décollage de la «Bat' » (à partir du plan 23).

\section{Ostinatos}

Le « réalisme » de l'image et des bruits, comme le montage image sur une musique pré-existante ${ }^{30}$, libère le compositeur d'un accompagnement à l'image trop servile ou trop explicite ${ }^{31}$ - même si, comme dans la partition d'Elfman, on peut affirmer que la

29 Voir l'entretien de Vasco Hexel avec Klaus Badelt (Hexel 2016, p. 41).

30 Cette musique pré-existante est appelée temp track pour " piste temporaire " : posée pour les besoins du montage image, elle sert ensuite de guide au compositeur qui en reprend le tempo et en retient généralement l'intention.

31 Cette volonté de coller à l'action pour la magnifier constituait l'un des rôles principaux de la musique dans les films d'action des années 1980, quand les trucages étaient plus complexes à réaliser en régime analogique qu'en régime numérique. Ainsi, dans le premier Superman, on voit le héros courir à côté d'un train... tandis que ses pieds ne touchent pas le sol! Pour Halfyard, " our potentially flaging suspension 
contrainte décuple l'imagination. Ainsi la musique de Zimmer peut-elle se développer sans entrave : c'est sans doute là l'un des secrets de son souffle épique si puissant ${ }^{32}$.

Déjà rencontré dans les deux scènes d'action précédentes, l'ostinato est sans conteste le procédé le plus récurrent - car le plus efficace - pour soutenir une action. Selon le musicologue Charles Rosen :

L'ostinato produit du mouvement à petit échelle, mais comme ce mouvement demeure inchangé, tout déplacement harmonique important est exclu. L'ostinato n'en est pas moins créateur d'instabilité, et il contribue au rythme global par l'effet de tension né de la répétition insistante : c'est un moyen simple de reconstituer l'effet de dissonance $[. .$.$] . Un ostinato qui accélère et en même temps augmente de volume est$ capable de produire une tension quasiment insoutenable (Rosen 1979, p. 62).

Qu'il soit rythmique, mélodique ou, comme c'est le plus souvent le cas, mélodico-rythmique, l'ostinato génère une tension qui est fonction de la durée de son maintien et du rythme harmonique. Nous proposons de comparer les ostinatos de deux nouvelles scènes d'action, s'appuyant chacune sur un ostinato dont nous allons détailler le maniement.

Lorsque Batman vient secourir son amie des griffes du Joker dans sa batmobile, Elfman commence par poser l'ostinato suivant (exemple 12) aux cordes (01:07:49) en même temps que flûtes, hautbois, cors et trompettes font entendre le motif de Batman :

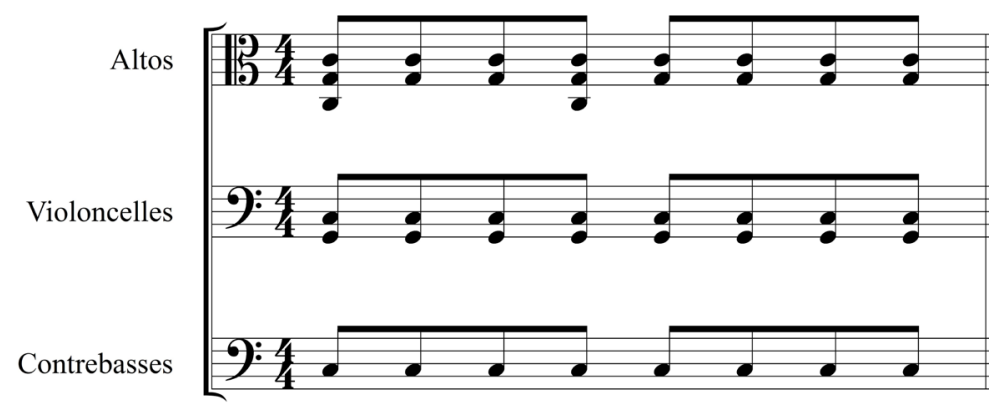

Exemple 12 : Danny Elfman, Batman. Transcription à partir du film ${ }^{33}$, 01:07:49.

Deux mesures plus tard, l'ostinato connaît un premier changement avec une transposition un ton plus bas (sib) avant de laisser place à un autre ostinato (exemple 13), fondé sur une oscillation au demi-ton harmonisée avec des accords parallèles (01:07:55-01:08:01) :

of disbelief is bolstered by the insistent heroic musical motif, which sonically reiterates Superman's superheroism, particularly in moments when it might be visually less convincing» (Halfyard 2013, p. 174).

32 Lehman (Lehman 2017) distingue également six emprunts (tropes) d'Hans Zimmer au répertoire savant occidental qui constituent la base de son "style épique " dans une forme de " minimalisme maximal» : l'« ostinato cathartique », la passacaille soutenue (ponderous passacaglia), le riff musical (marcato motto), l'écriture chorale résultante (consequential chorales) - résultant de la proximité des différentes voix dans le cadre d'harmonies simples (qui proviennent du jeu pianistique de Zimmer plutôt que d'un manuel d'orchestration) -, le thème teuton et l'hymne réduite (abstract anthem).

33 Nos transcriptions musicales de la partition d'Elfman s'appuient partiellement sur les relevés de Solis 2013, p. 66-72. 


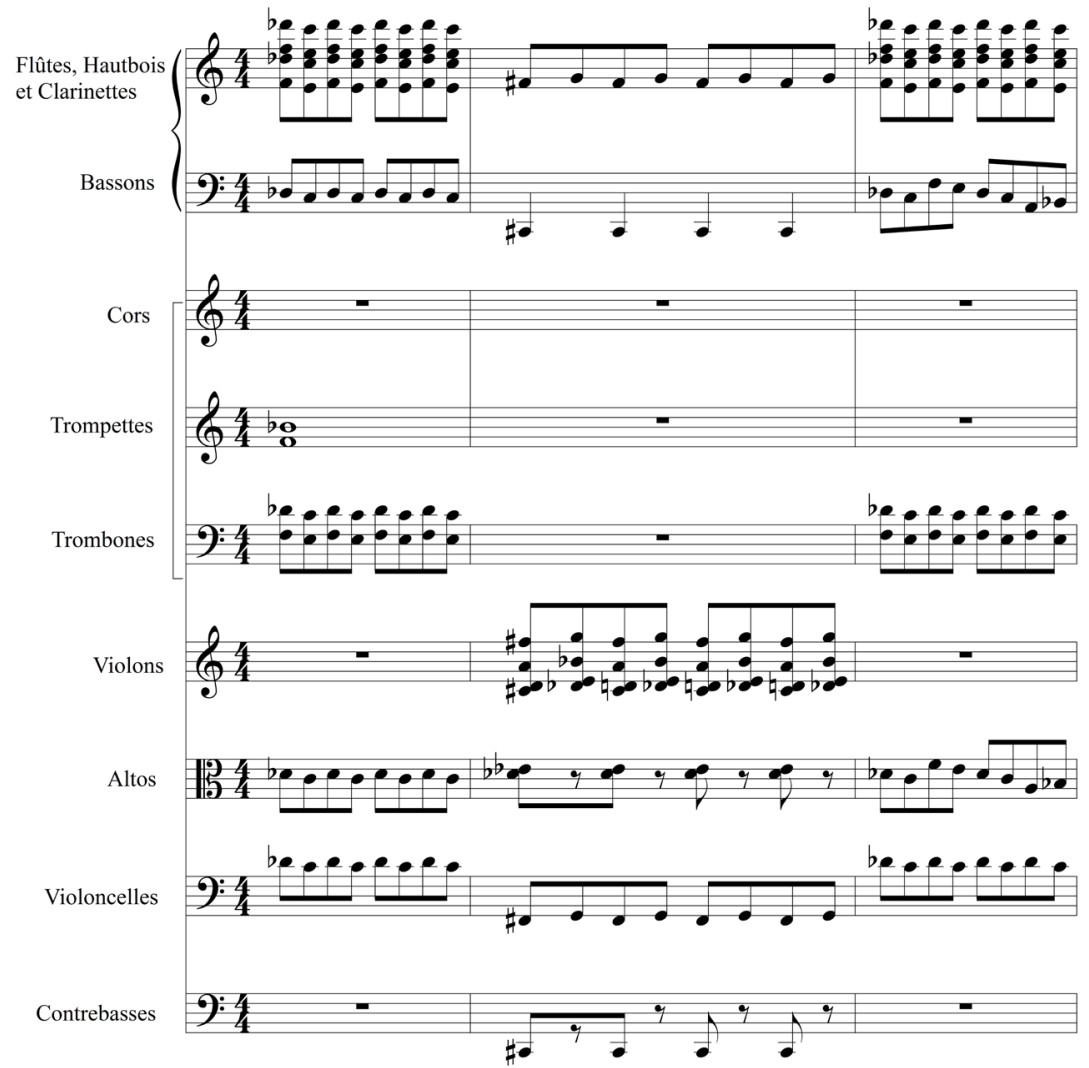

Exemple 13 : Danny Elfman, Batman. Transcription à partir du film, 01:07:55-01:08:01.

Le rythme à la croche persiste, mais les mesures de l'exemple 13 sont construites selon un dispositif antiphonal faisant dialoguer deux dispositifs instrumentaux. À partir de la mesure suivante (contrebasses, exemple 14), l'oscillation n'est plus au demi-ton, mais au ton complet ; des motifs de fanfare aux vents viennent troubler l'aspect répétitif de l'ensemble.

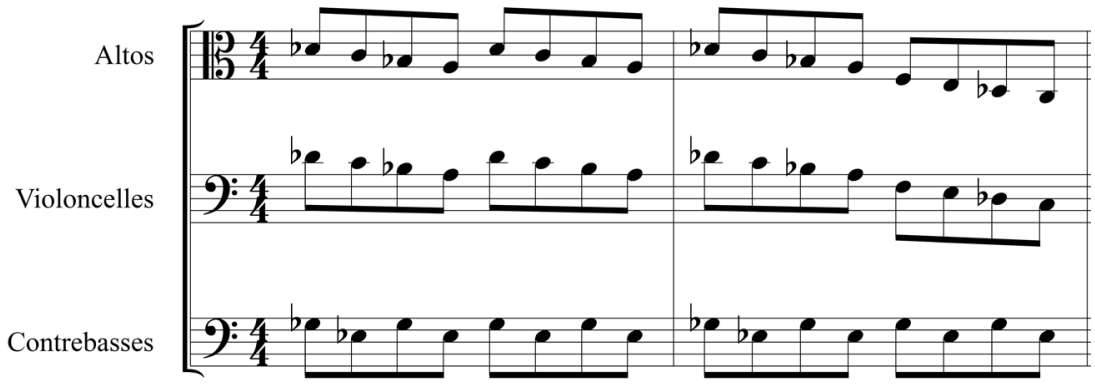

Exemple 14 : Danny Elfman, Batman. Transcription à partir du film, 01:08:02-01:08:04.

Le motif de Batman revient ensuite à divers vents, tandis que les cordes aèrent le dispositif par leur absence une mesure sur deux (exemple 15) ; on retrouve l'oscillation au demi-ton du début aux cordes. 


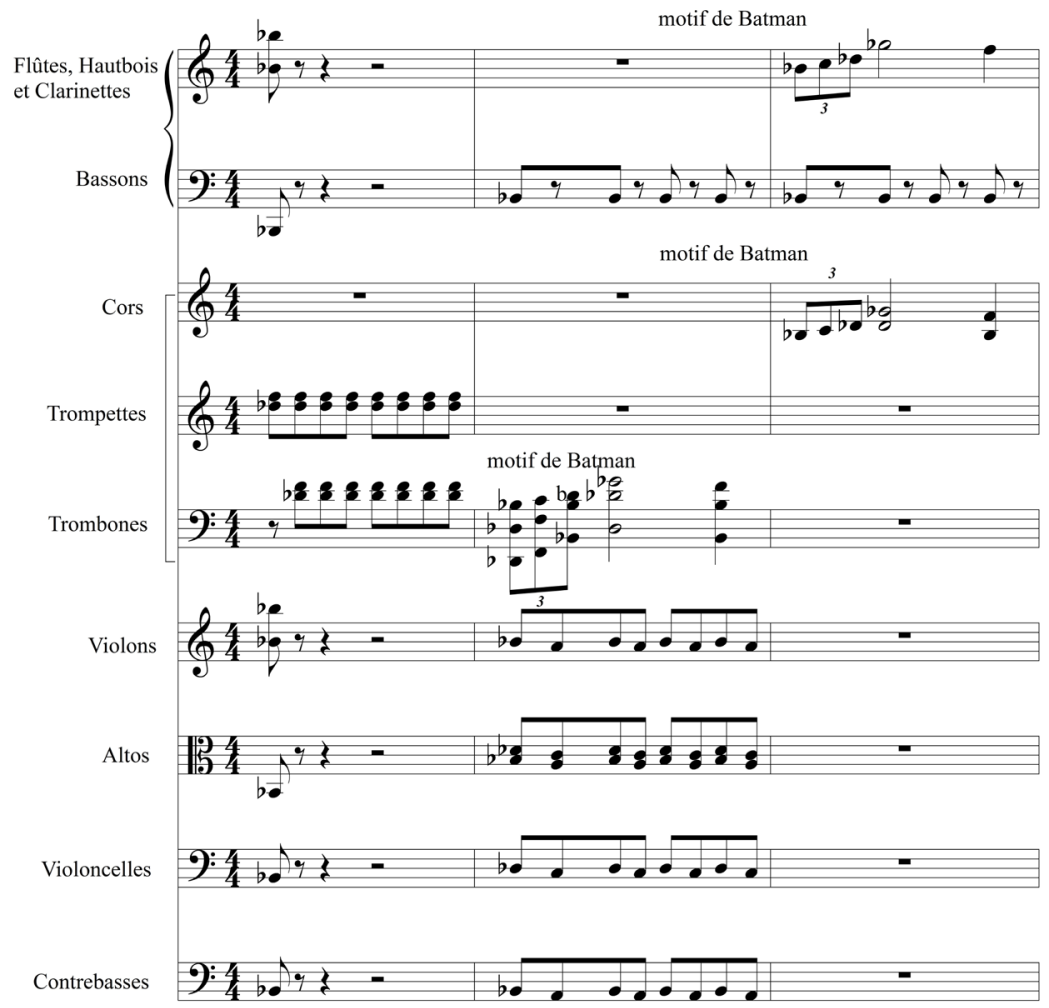

Exemple 15: Danny Elfman, Batman. Transcription à partir du film, 01:08:05-01:08:09.

À la fin du cue, Elfman redonne deux mesures avec l'ostinato aux cordes avant d'y mettre un terme définitivement à partir de la mesure à 3/4 (exemple 16).

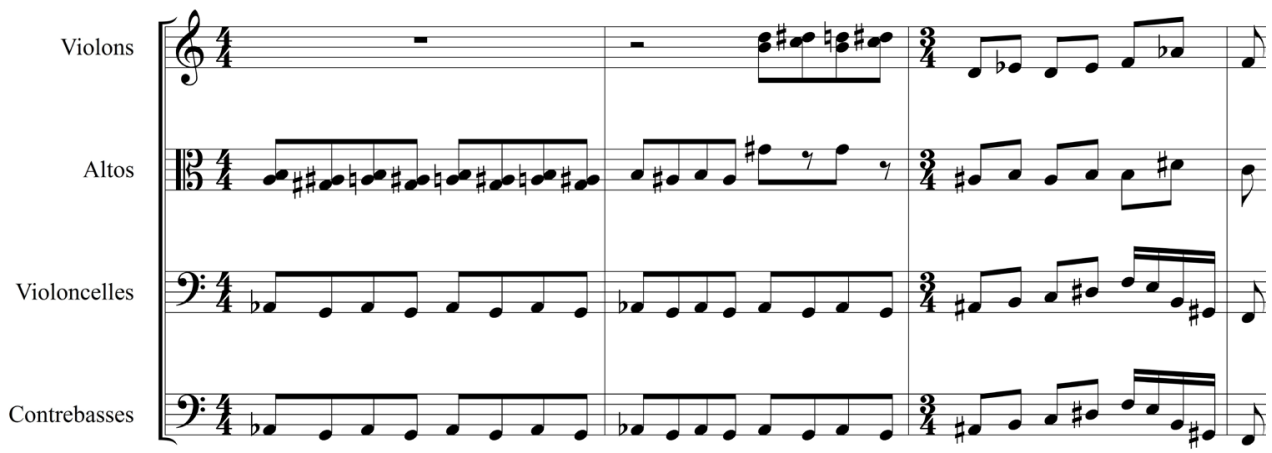

Exemple 16 : Danny Elfman, Batman. Transcription à partir du film, 01:08:12-01:08:14.

Cette courte analyse suffit ici à montrer l'extrême vivacité de l'écriture d'Elfman, avec un rythme harmonique extrêmement rapide et des dispositifs instrumentaux toujours renouvelés - au minimum toutes les deux mesures -, afin de souligner les césures visuelles.

Nous allons à présent observer le maniement d'un ostinato dans le cadre d'une scène d'action chez Zimmer, au moment où Kyle tue Bane alors que celui-ci s'apprêtait à assassiner Batman. Notons d'ores-et-déjà la longueur de cet ostinato qui couvre cinq minutes d'action, alors même que le passage d'Elfman analysé ci-dessus ne se maintenait que quelques secondes. À 02:22:28, un ostinato mélodico-rythmique sur pédale se met en place (exemple 17) : 


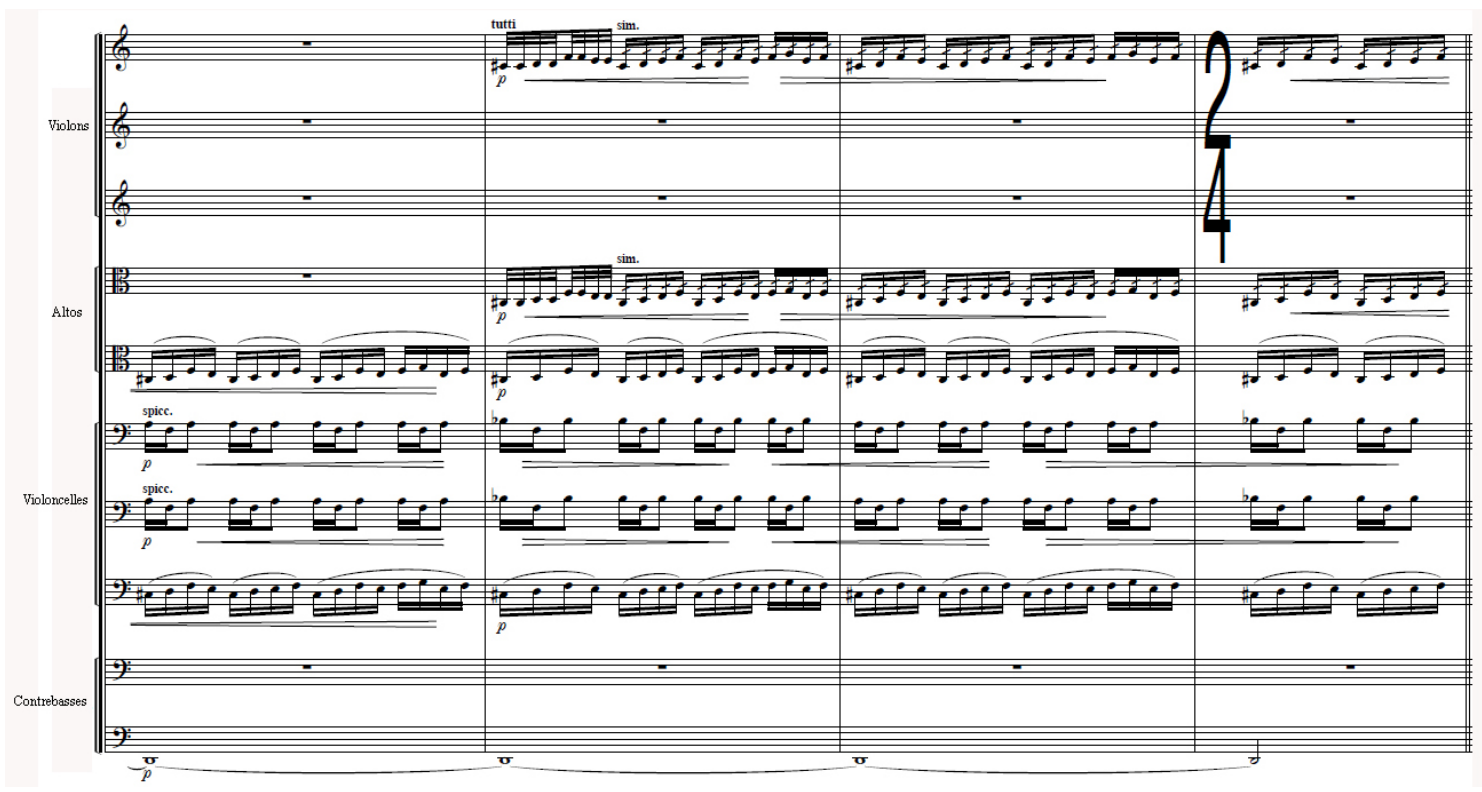

Exemple 17: Hans Zimmer, The Dark Knight Rises. Transcription à partir du film, 02:22:28-02:22:38.

On notera la complexité de l'ostinato - ce que Lehman appelle le « minimalisme maximal $»^{34}$ (Lehman 2017, p. 30) -, obtenue à l'aide de la superposition de trois strates dotées de motifs mélodico-rythmique différents avec des pupitres de cordes divisés ; ces cordes jouent tantôt spiccato tantôt marcato avec des accents, conférant à l'ensemble une sonorité rugueuse et grouillante. Cet ostinato sert de trame orchestrale à des citations du motif de Batman - tierce mineure ascendante - ou du thème de Selina Kyle (voir exemple 18 aux violons ${ }^{35}$ ), selon que l'on voit l'un ou l'autre à l'écran ; observons que ces citations ne provoquent aucun changement de pulsation ni de texture orchestrale - cette dernière se trouve seulement un peu allégée dans les graves lors de la citation de l'« ostinato identifiant » de Kyle :

34 "While tonally the music is static, the enormous accretion of sound indicates that surface simplicity is in service of maximal ends: maximum textural complexity and detail, maximum volume, maximum emotive power. »

35 Une autre récurrence de ce thème est indiquée entre crochet dans le parcours harmonique de la figure 3 à 24:55. 


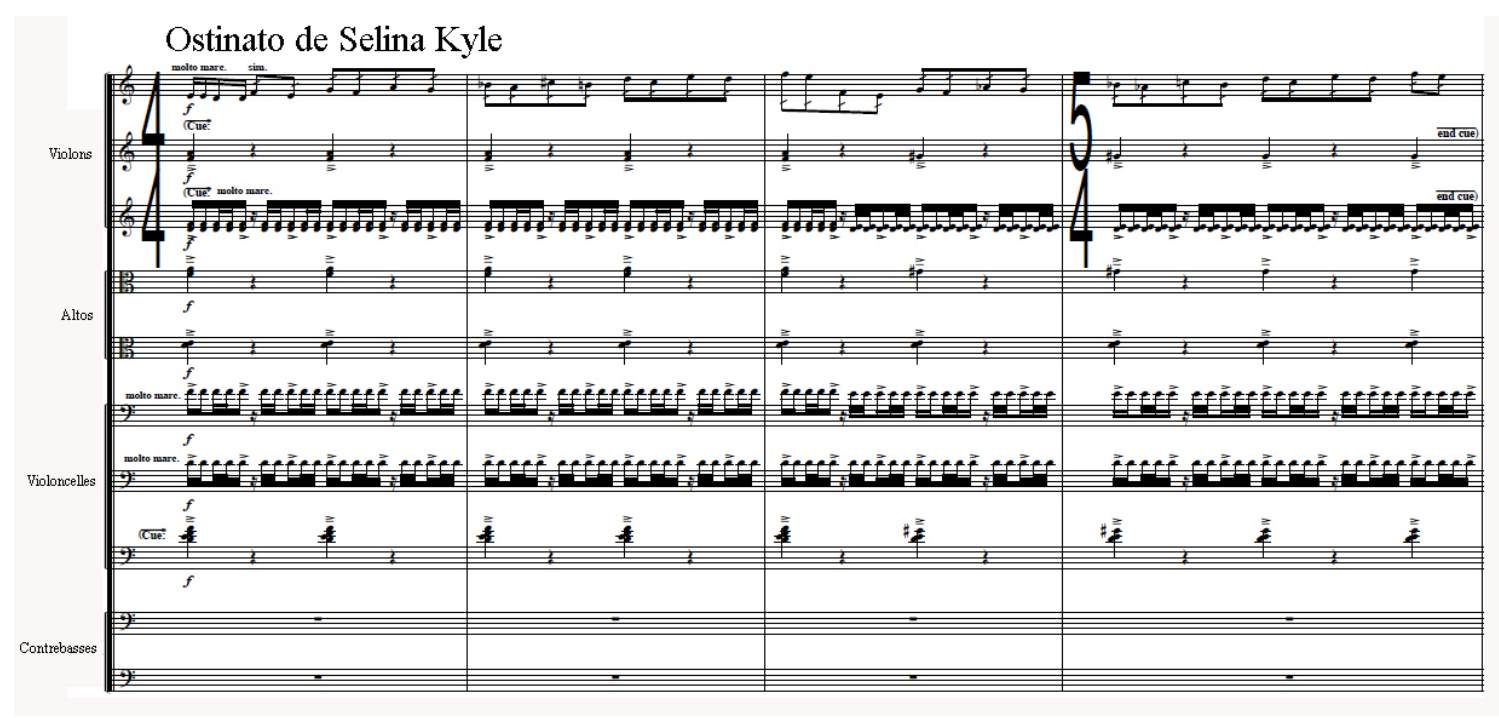

Exemple 18: Hans Zimmer, The Dark Knight Rises. Transcription à partir du film, 02:22:41-02:22:47.

Le jeu sur la quantité de strates (« stem scoring») - déjà observé précédemment dans la scène où Batman vient secourir Selina Kyle - permet d'amenuiser ou d'amplifier à loisir l'intensité musicale de l'ostinato en autant de variations musicales adaptées au propos, un dispositif commode pour soutenir de longues scènes. Cette stratégie - qui relève finalement autant du mixage (ou plutôt du pré-mixage) que de la composition - se trouve complétée par d'autres procédés compositionnels, impliquant pédales harmoniques et jeu sur le rythme harmonique. L'ostinato de Batman, principalement polarisé sur ré mineur, accompagne la bataille finale (02:22:22-02:27:21) ; elle s'achève par le meurtre de Bane et la libération de Gotham de la menace de la bombe. Notre analyse se concentre sur la dernière partie (02:24:17-02:27:21). L'ostinato en lui-même est extrêmement répétitif, principalement axé sur les notes ré et $f a$ (tierce mineure de Batman, presque continuellement présent à l'écran). Lehman qualifie ce type d'ostinato de « cathartique»:

The basis for these ostinati are catchy, melodically simple gestures that are tailored for their amenability to repetition. The obsessive reiteration structures ensure that the listeners can be led blissfully along, never worrying that the score will lead them on an unexpected course, that any unexpected musical tension might threaten to break the spell. This gripping quality derives from the small size - and thus low cognitive load - of the repeated units (ibid., p. 29).

Les variations viennent principalement de la gestion harmonique, résumée par la figure 4 qui met en regard les harmonies et le nombre de plans ${ }^{36}$. Chaque case correspond à une mesure de quatre temps dans un tempo que nous avons estimé à 80 à la noire (pulsation isochrone) ; ces mesures à quatre temps se succèdent à peu près

36 Le nombre de plans est ici supérieur au nombre de plan réel car notre segmentation a pris pour référence la mesure musicale ; le nombre de plan indiqué correspond au nombre de plans différents contenus dans une mesure. 
toutes les trois secondes. Les cases grisée correspondent à des pédales harmoniques (en ré mineur) s'étalant sur un nombre un peu plus important de mesures (se référer au temps). ces pédales sont l'occasion d'une accélération spectaculaire du nombre de plans dans une forme de complémentarité kinesthésique inversement proportionnelle, une stase harmonique correspondant à une augmentation du nombre de plans. On constate que ces pédales harmoniques durent de moins en moins longtemps à mesure que l'on s'approche de la fin de la séquence $(25 \mathrm{sec}$., puis $22 \mathrm{sec}$., $17 \mathrm{sec}$. et enfin $13 \mathrm{sec}$.). L'imminence du dénouement (tension liée à l'écoulement du temps qui rapproche inéluctablement 1'explosion de la bombe) se fait aussi sentir dans la diminution du rythme harmonique qui voit se succéder des accords de manière de plus en plus rapide. Zimmer commence par installer un rythme harmonique à la ronde (nous n'avons pas pris en considération les changements de renversements qui nous semblent plutôt ressortir d'un chant à la basse). Alors que le nombre de plans reste stable pendant toute la séquence (entre deux et trois plans par mesure), le rythme harmonique diminue à partir de 02:26:11, même si on trouve encore quelques harmonies à la ronde ; à partir de 02:26:45, le rythme harmonique est définitivement passé à la blanche. Conjugué à l'augmentation du nombre de plans (3 voire 4 par mesure), la séquence se trouve précipitée vers sa fin, actée par un saut d'octave au cor sur une harmonie de septième majeure avec quinte diminuée (02:27:21), geste mélodico-harmonique qui conclut toutes les actions finales de la trilogie ${ }^{37}$, interrompant l'ostinato alors que le camion contenant la bombe effectue une chute spectaculaire qui le brise.

\begin{tabular}{|l|l|l|l|l|l|l|l|l|l|}
\hline Tps & $02: 24: 17$ & $02: 24: 20$ & $02: 24: 23$ & $02: 24: 26$ & $02: 24: 30$ & $02: 24: 33$ & $02: 24: 36$ & $02: 24: 39$ & $02: 24: 42$ \\
\hline Harm. & Dm & $\mathrm{C}$ & $\mathrm{Dm}$ & $\mathrm{C}$ & $\mathrm{Dm}$ & $\mathrm{C}$ & $\mathrm{Dm}$ & $\mathrm{Am}$ & $\mathrm{Dm}$ \\
\hline Plans & 2 & 2 & 2 & 2 & 3 & 3 & 1 & 1 & 1 \\
\hline
\end{tabular}

\begin{tabular}{|l|l|l|l|l|l|l|l|l|l|}
\hline $02: 24: 46$ & $02: 24: 49$ & $02: 24: 52$ & $02: 24: 55$ & $02: 25: 20$ & $02: 25: 23$ & $02: 25: 26$ & $02: 25: 48$ & $02: 25: 51$ & $02: 25: 54$ \\
\hline $\mathrm{Am}$ & $\mathrm{Dm}$ & $\mathrm{Am}$ & $\mathrm{Dm}[\mathrm{Kyle}]$ & $\mathrm{CH}$ & $\mathrm{CH}$ & $\mathrm{Dm}$ & $\mathrm{AM} / \mathrm{C} \#$ & $\mathrm{AM} / \mathrm{C} \#$ & $\mathrm{Dm}$ \\
\hline 1 & 2 & & 22 & 3 & & 15 & 2 & & 10 \\
\hline
\end{tabular}

\begin{tabular}{|l|l|l|l|l|l|l|l|l|l|l|l|}
\hline $02: 26: 11$ & \multicolumn{2}{|l|}{$02: 26: 14$} & $02: 26: 17$ & $02: 26: 30$ & $02: 26: 33$ & $02: 26: 36$ & $02: 26: 39$ & $02: 26: 42$ \\
\hline $\mathrm{C}$ & $\mathrm{Am}$ & $\mathrm{Bb}$ & $\mathrm{C}$ & $\mathrm{Dm}$ & $\mathrm{Fm} 7$ & $\mathrm{Am}$ & $\mathrm{F}$ & $\mathrm{Cm}$ & $\mathrm{Ab}$ & $\mathrm{Dm}$ & $\mathrm{Dm}$ \\
\hline 2 & & 2 & & 9 & 3 & 2 & 3 & & 4 & 2 \\
\hline
\end{tabular}

\begin{tabular}{|c|c|c|c|c|c|c|c|c|c|c|c|}
\hline \multicolumn{2}{|c|}{$02: 26: 45$} & \multicolumn{2}{|c|}{$02: 26: 49$} & \multicolumn{2}{|c|}{$02: 26: 52$} & \multicolumn{2}{|c|}{$02: 26: 55$} & \multicolumn{2}{|c|}{$02: 26: 58$} & \multicolumn{2}{|c|}{ 02:27:01 } \\
\hline $\mathrm{Dm}$ & $\mathrm{Gm}$ & $\mathrm{Dm} / \mathrm{A}$ & $\mathrm{Gm}$ & $\mathrm{F} / \mathrm{A}$ & $\mathrm{Gm} / \mathrm{Bb}$ & $\mathrm{Am} / \mathrm{C}$ & $\mathrm{A} / \mathrm{CH}$ & Dm & $\mathrm{Bb}$ & $\mathrm{Fm}$ & $\mathrm{Db}$ \\
\hline 2 & & 2 & & 2 & & 1 & & 3 & & 4 & \\
\hline
\end{tabular}

\begin{tabular}{|l|l|l|l|l|l|l|l|l|}
\hline 02:27:04 & \multicolumn{2}{|l|}{$02: 27: 07$} & $02: 27: 10$ & \multicolumn{2}{|l|}{$02: 27: 14$} & \multicolumn{2}{|l|}{$02: 27: 17$} \\
\hline $\mathrm{Dm}$ & $\mathrm{Bb}$ & $\mathrm{Fm}$ & $\mathrm{Db}$ & $\mathrm{Dm}$ & $\mathrm{Dm}$ & $\mathrm{Gm}$ & $\mathrm{Dm}$ & $\mathrm{Cm}$ \\
\hline 4 & & 4 & & 3 & 3 & & & \\
\hline
\end{tabular}

Figure 4 : Hans Zimmer, The Dark Knight Rises, trame harmonique du combat final et nombre de plans Transcription à partir du film, 02:24:17-02:27:21.

37 Voir à 02:03:53 lorsque Batman laisse Ra's al Ghul se tuer dans la rame de métro dans Batman Begins et à 02:07:44 lorsque Batman lance le Joker (Heath Ledger) dans le vide dans The Dark Knight. 
Extrait vidéo 9: Christopher Nolan, The Dark Knight Rises, combat final, 02:24:17-02:27:21

(C) Warner Home Video.

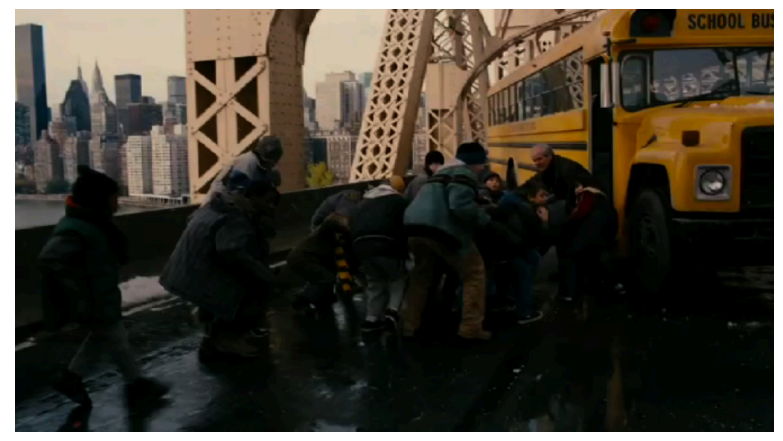

La puissance de cette séquence provient tant de la mise en scène " réaliste " et spectaculaire de Nolan, que d'une kinesthésie audiovisuelle qui repose sur le rythme du montage et les effets conjugués de la permanence de l'ostinato mélodico-rythmique, des pédales harmoniques et de la diminution du rythme harmonique.

Beaucoup d'éléments rattachent finalement les conceptions d'Elfman et de Zimmer, par-delà la vingtaine d'années qui séparent la fabrication des deux films : le rôle symbolique des thèmes, la présence presque continue de la musique, la recherche de synchronismes, l'emploi d'ostinatos. Un examen détaillé révèle toutefois de très sensibles différences. Quand Elfman identifie musicalement son super-héros avec un seul motif - au point de concevoir une partition quasiment mono-thématique -, Zimmer conçoit, pour chacun des personnages, au moins deux matériaux thématiques (un thème et un ostinato), voire un matériau multiple pour Batman ; plus nombreux, les thèmes de Zimmer sont aussi moins caractérisés musicalement. Halfyard y voit un signe des temps : les musiques super-héroïques servent désormais moins à mettre en valeur l'héroïsme des actions que la part d'humanité et la complexité psychologique de ces nouveaux super-héros, " as soldiers on the front line in wars against terror $»^{38}$.

Mais c'est surtout dans le rapport rythmique à l'image que les différences s'accusent. L'évolution de la technologie a permis à Nolan, Zimmer, King et Wesson de travailler une " narration sonore » qui intègre la musique dans un continuum avec les bruits. L'extrême précision de ces derniers a pour conséquence une émancipation de la musique par rapport au renforcement systématique des changements de plans ou des mouvements de l'action - un renforcement dont on a pu tout de même observer l'inventivité chez Elfman. Alors que ce dernier élabore un tissu musical discontinu et varié, avec de courts ostinatos permettant un synchronisme serré, Zimmer adopte une stratégie compositionnelle privilégiant la continuité avec des ostinatos longs, dont les principales variations proviennent du "stem scoring » et du rythme harmonique; moins précise à l'image, cette écriture privilégie une plus grande continuité musicale qui enveloppe les images en leur conférant un dynamisme et une ampleur exceptionnels. Ces ostinatos sont d'autant plus remarquables qu'ils continuent à porter l'identité des protagonistes

38 Halfyard 2013, p. 192. Manohla Dargis propose le terme de "postheroic superhero movie " pour qualifier ces films (Dargis 2008). 
au cour des scènes d'action grâce à la technique des " ostinatos identifiants » qui remplacent les « fameux leitmotive » trop tapageurs ${ }^{39}$ (Halfyard 2013, p. 182).

C'est finalement tout l'art du compositeur de musique de blockbusters super-héroïques que de soutenir une scène d'action tout en l'associant musicalement à ses protagonistes - des protagonistes dont les thèmes musicaux contribuent si fortement à forger les identités. Dans sa récente partition pour Justice League (Z. Snyder, 2017), Danny Elfman réemploie un certain nombre de thèmes de super-héros (le thème de Superman de John Williams, son propre thème pour Batman) et va jusqu'à utiliser exclusivement le thème de Wonderwoman - originellement joué par la violoncelliste Tina Guo et composé pour Batman versus Superman (Z. Snyder, 2016, musique de Hans Zimmer et Junkie XL) - pour soutenir symphoniquement la jeune amazone alors qu'elle couvre les otages de balles qui pleuvent sur eux (10:36-10:38 puis 10:4010:42). Preuve de l'influence incontestable de Zimmer sur l'industrie musicale cinématographique, Elfman a infléchi son écriture vers une utilisation plus importante des ostinatos ${ }^{40}$ avec un instrumentarium pléthorique incluant cuivres, percussions et cordes graves. Toutefois, les marqueurs elfmanniens - enchaînements harmoniques par couleur, extrême vivacité rythmique, utilisation du célesta et des chœurs demeurent présents, démontrant magistralement que musique d'action ne rime pas avec uniformisation.

\section{BIBLIOGRAPHIE}

Anonyme (2012), Soundworks Collection, The Sound and Music of The Dark Knight Rises, http://soundworkscollection.com/videos/darkknightrises, consulté le 20 mars 2017.

Boucher, Geoff (2008), "Christopher Nolan on "Dark Knight" and its Box-Office Billion. "It's Mystifying to Me" ", Hero complex, http://herocomplex.latimes.com/movies/christopher-nol/, consulté le 26 mars 2018.

Brooker, Will (2012), Huntling the Dark Knight: Twenty-First Century Batman, Londres et New York, I. B. Tauris.

Brown, Royal S. (1994), Overtones and Undertones: Reading Film Music, Los Angeles et Berkeley, University of California Press.

39 "In the era of digital technology, however, the action has become so thoroughly convincing that it does not need music to bolster its effectiveness. Heroic themes might, in fact, detract from the slick hyperrealism of a Superman who moves through the air like a swimmer through water, capable of rolling and turning, as he does when he rolls over onto his back while flying down a city street and blasts falling debris to harmless dust with heat rays from his eyes. [...] I argue that there is no need for music to plaster over the cracks in his credibility. Despite the often continuous music, the cues for these sequences give pace to the action but do not draw attention to themselves by trumpeting the famous leitmotifs, and our attention can remain focused on the action as spectacle in its own right. There is no need for music to cast a glamour of believability over it » (Halfyard 2013, p. 182).

40 Voir par exemple le cue " Hero's Theme ». Remarquons qu'Elfman y superpose une variation de son thème de Batman ( $f a$-sol-lab-réb-do) avec la tierce mineure ascendante (fa-lab) du thème de Batman de Zimmer - qui sert précisément d'ostinato. 
Burlingame, Jon (2014), "Billion-Dollar Composer. For Hans Zimmer, "The Real Risk is Playing It Safe" ", Variety (7 mai), http://variety.com/2014/music/features/20-billion-dollar-composer-forhanszimmer-the-real-risk-is-playing-it-safe-1201173698/, consulté le 21 mars 2018.

Chion, Michel (1995), La musique au cinéma, Paris, Fayard.

Colburn, Michael J. (2004), "John Williams Returns to Bands Where He Began 50 Years Ago », The Instrumentalist, vol. 58, no 11, p. 16.

Dargis, Manohla (2008), "Showdown in Gotham Town ", New York Times (juillet), http://www. nytimes.com/2008/07/18/movies/18knig.html, consulté le 21 mars 2018.

Halfyard, Janet (2004), Danny Elfman's Batman, Lanham, Londres et New York, Scarecrow Press.

Halfyard, Janet K. (2013), «Cue the Big Theme? The Sound of the Superhero », dans John Richardson, Claudia Gorbman, and Carol Vernallis (dir.), The Oxford Handbook of New Audiovisual Aesthetics, New York, Oxford University Press, p. 170-193.

Hexel, Vasco (2016), Hans Zimmer and James Newton Howard's The Dark Knight, Lanham, Rowman \& Littlefield.

Hyman, Dan (2012), «Dark Knight Rises'Composer on Creating Gotham City's Ominous Sonic Landscape», Rolling Stone (juillet), https://www.rollingstone.com/music/news/dark-knight-rises-composer-oncreating-gotham-citys-ominous-sonic-landscape-20120721, consulté le 4 avril 2018.

Jess-Cooke, Carolyn (2009), Film Sequels: Theory and Practice from Hollywood to Bollywood, Edinburgh, Edinbugh University Press.

Kerins, Mark (2015), «The Modern Entertainment Marketplace, 2000-Present », dans Kathryn Kalinak (dir.), Sound, Dialogue, Music, and Effects, Londres et New York, I.B. Tauris, p. 133-155.

Langlois, Philippe (2012), Les cloches d'Atlantis : Musique électroacoustique et cinéma. Archéologie et histoire d'un art sonore, Paris, Editions MF.

Lehman, Frank (2017), «Manufacturing the Epic Score, Hans Zimmer and the Sounds of Signifiance », dans Stephen C. Meyer (dir.), Music in Epic Film, Listening to Spectacle, New York et Londres, Routledge.

Miceli, Serge et Morricone, Ennio (2013), Composing for the Cinema, New York, Scarecrow Press.

Page, Edwyn (2007), Gothic Fantasy: The Films of Tim Burton, Londres, Marion Boyars Publishers.

Pigeon, Ted (2008), "Sounds of the Knight ", blog (juin), http://tedpigeon.blogspot.fr/2008/06/ sounds-of-knight.html, consulté le 22 mars 2018.

Radish, Christina (2011), "Composer Hans Zimmer Talks About Crowd Sourcing Chants for the Score of The Dark Knight Rises», Collider.com (décembre), http://collider.com/hans-zimmerdark-knight-rises-interview/, consulté le 14 septembre 2018.

Rosen, Charles (1979), Schoenberg, Paris, Les éditions de Minuit.

Rossi, Jérôme (2011), " Le dynamisme harmonique dans l'écriture filmique de John Williams », dans Alexandre Tylski (dir.), John Williams, Un alchimiste musical à Hollywood, Paris, L'Harmattan, 2011, p. 113-140.

Sergi, Gianluca (2006), "In Defence of Vulgarity: The Place of Sound Effects in the Cinema ", Scope. An online journal of film studies, $\mathrm{n}^{\circ} 5$ (juin), https://www.nottingham.ac.uk/scope/documents/2006/ june-2006/sergi.pdf, consulté le 9 avril 2018.

Solis, Israel (2013) (Re)creating a Hero's Narrative through Music: Different Musical Lanscape in Six Live Action Batman Films, thèse de doctorat, Université d'Arizona.

Tagg, Philip (1982), « Theory and Method », Popular Music, vol. 2, p. 37-67.

Tagg, Philip (1989), "An Anthropology of Stereotypes in TV Music? ", Swedish Musicological Journal, vol. 71, p. 19-42, http://www.tagg.org/articles/xpdfs/tvanthro.pdf, consulté le 21 mars 2018.

Wayner, Alex (2014), Batman as Mythic Figure in Comic and Film, Jefferson, McFarland and Company. 